\title{
Recent applications and strategies in nanotechnology for lung diseases
}

\author{
Wenhao Zhong ${ }^{1, \S}$, Xinyu Zhang ${ }^{1, \S}$, Yunxin Zeng ${ }^{1}$, Dongjun $\operatorname{Lin}^{1}(\varangle)$, and Jun $\mathrm{Wu}^{1,2}(\varangle)$ \\ ${ }^{1}$ Department of Hematology, The Seventh Affiliated Hospital, Sun Yat-sen University, Shenzhen 518107, China \\ ${ }^{2}$ Key Laboratory of Sensing Technology and Biomedical Instrument of Guangdong Province, School of Biomedical Engineering, Sun Yat-sen \\ University, Guangzhou 510006, China \\ ${ }^{\S}$ Wenhao Zhong and Xinyu Zhang contributed equally to this work.
}

(C) Tsinghua University Press and Springer-Verlag GmbH Germany, part of Springer Nature 2020

Received: 15 September 2020 / Revised: 11 October 2020 / Accepted: 11 October 2020

\begin{abstract}
Lung diseases, including COVID-19 and lung cancers, is a huge threat to human health. However, for the treatment and diagnosis of various lung diseases, such as pneumonia, asthma, cancer, and pulmonary tuberculosis, are becoming increasingly challenging. Currently, several types of treatments and/or diagnostic methods are used to treat lung diseases; however, the occurrence of adverse reactions to chemotherapy, drug-resistant bacteria, side effects that can be significantly toxic, and poor drug delivery necessitates the development of more promising treatments. Nanotechnology, as an emerging technology, has been extensively studied in medicine. Several studies have shown that nano-delivery systems can significantly enhance the targeting of drug delivery. When compared to traditional delivery methods, several nanoparticle delivery strategies are used to improve the detection methods and drug treatment efficacy. Transporting nanoparticles to the lungs, loading appropriate therapeutic drugs, and the incorporation of intelligent functions to overcome various lung barriers have broad prospects as they can aid in locating target tissues and can enhance the therapeutic effect while minimizing systemic side effects. In addition, as a new and highly contagious respiratory infection disease, COVID-19 is spreading worldwide. However, there is no specific drug for COVID-19. Clinical trials are being conducted in several countries to develop antiviral drugs or vaccines. In recent years, nanotechnology has provided a feasible platform for improving the diagnosis and treatment of diseases, nanotechnology-based strategies may have broad prospects in the diagnosis and treatment of COVID-19. This article reviews the latest developments in nanotechnology drug delivery strategies in the lungs in recent years and studies the clinical application value of nanomedicine in the drug delivery strategy pertaining to the lung.
\end{abstract}

\section{KEYWORDS}

lung diseases, drug delivery, nanoparticles

\section{Introduction}

As an important organ of the respiratory system, the lung performs a key role in human life. The lung has unique anatomical characteristics, and its special physiological environment has resulted in the increasing focus on lung drug delivery strategies in current clinical research [1].

\subsection{Anatomical features, barriers, and deposition in pulmonary airways}

The lungs are primarily composed of bronchi, small bronchi, alveolar tubes, alveoli, and pulmonary blood vessels. The pulmonary blood vessels primarily include pulmonary arteries, pulmonary veins, and microvessels. Besides, there is the large alveolar surface area in each lung. When treating lung diseases, therapeutic drugs are usually required to overcome the following two major lung barriers. (1) Mechanical barrier: The diseased state of the lungs (such as infection) can cause bronchoconstriction or excessive mucus secretion, resulting in narrowing of the airway. In addition, the lung mucosa cilia can remove particles deposited in the airway. These are some of the manifestations of the mechanical barrier of the lungs [2]. (2) Chemical and immunological barriers: The chemical and immune barriers of the lungs are primarily composed of surfactants, proteolytic enzymes, and alveolar macrophages [3]. They all have an influence on the working of the lungs; moreover, overcoming the adverse effects of these obstacles is conducive to improving the efficacy of lung therapy drugs. After the drug enters the lung, it is deposited. The deposition of the drug involves a significantly complicated process, which is controlled by factors such as the location of the deposition, deposition rate, and particle size of the drug. The deposition of drugs in the lungs is primarily achieved through impaction, sedimentation, interception, diffusion, absorption, and so on [4].

\subsection{Common lung diseases}

\subsubsection{Lung cancer}

Lung cancer originates in the bronchial mucosal epithelium and alveoli. It is one of the malignant tumors with the highest morbidity and mortality rates worldwide, and it seriously endangers human health [5]. 


\subsubsection{Cystic fibrosis (CF)}

$\mathrm{CF}$ is caused by mutations in the transmembrane conductance regulation gene of CF. It is primarily manifested as endocrine and exocrine gland dysfunctions, such as mucosal gland hyperplasia and thickened secretions. Lung diseases are significantly serious and common. The pathogenesis is primarily the obstruction of the bronchus by viscous secretions and secondary infections. Early clogging of viscous secretions can cause atelectasis, secondary infections, repeated infections, and gradually result in extensive lung fibrosis and obstructive emphysema; finally, it can lead to pulmonary hypertension, respiratory failure, and pulmonary heart disease. The primary pathogen of CF in combination with lung infection is Pseudomonas aeruginosa (PA). Once the respiratory tract of a $\mathrm{CF}$ patient is invaded by $\mathrm{PA}$, this infection will recur and cause persistent lung damage. Currently, it is believed that the cause of recurrent infections is not only the obstruction of respiratory secretions, but also the formation of a biofilm by PA in the lungs, which leads to bacterial resistance [6].

\subsubsection{Chronic obstructive pulmonary disease (COPD)}

Chronic obstructive pulmonary disease (COPD) is a common lung disease that has a long refractory period and high mortality rate. COPD is a preventable and treatable disease characterized by persistent airflow limitation. The primary clinical symptoms are chronic cough, expectoration, and asthma. It is worth noting that after inhaling bronchodilators, when the ratio of forced expiratory volume in $1 \mathrm{~s}$ (FEV1) to forced vital capacity $<70 \%$, it indicates that there is continuous airflow limitation [7]. Currently, antibiotics, corticosteroids, $\beta$-agonists, and bronchodilators are commonly used in the treatment of COPD.

\subsubsection{Pulmonary tuberculosis}

Tuberculosis is widespread worldwide and is a global public health and social issue. Pulmonary tuberculosis is the most important type of tuberculosis; $90 \%$ of the pathogenic bacteria of tuberculosis is the Mycobacterium tuberculosis (Mtb). Pulmonary tuberculosis patients are the primary source of the infection, especially those who are positive for sputum bacteria, which are predominantly transmitted through droplets, i.e., by expelling particles containing tuberculosis into the air.

\subsubsection{Pneumonia}

Pneumonia is primarily a lung disease caused by a variety of pathogens. Common pathogens include fungi, bacteria, and viruses. Bacterial pneumonia is the most common pneumonia and one of the most common infectious diseases. The emergence and development of antibiotics and viral vaccines have significantly reduced the mortality rate of pneumonia; however, in recent years, antibiotic resistance has become a significant challenge in the treatment of pneumonia.

\subsubsection{Bronchial asthma}

Bronchial asthma is a common chronic inflammatory disease of the airway. The chronic attack leads to the occurrence and development of airway hyperresponsiveness. Clinical manifestations are recurrent shortness of breath, wheezing, coughing, chest tightness, and so on. Bronchial asthma attacks often occur at night and/or early in the morning, accompanied by reversible airflow acceleration.

\subsection{Strategy of pulmonary drug delivery}

Drugs for the treatment of lung diseases primarily enter the human body through intravenous injection, oral administration, or inhalation to achieve therapeutic effects (Fig. 1). The lung can be used as an ideal target for local administration owing to its unique anatomical and physiological characteristics [8-10].

Oral drug therapy is used to treat respiratory diseases. Because of the large surface area of the intestinal epithelium and easy administration, this method can encourage patients to consume drugs according to plan $[10,11]$. However, there are several defects in the enteral route of administration. For example, the barrier effect caused by the epithelial barrier can inhibit the absorption of hydrophilic drugs; moreover, it is difficult for therapeutic drugs to effectively treat the lesion. Moreover, the degradation of drugs during gastrointestinal transport and nonselective distribution of drug carriers can result in toxic effects. The above obstacles can be resolved in the parenteral (usually intravenous) route of administration $[12,13]$.

Intravenous administration for the treatment of respiratory diseases can bypass the step of penetrating the mucosal barrier, which is still a challenge in the inhalation route of administration. However, the intravenous route is an invasive route of administration and results in inconvenience to the patient, higher medical costs, and side effects of treatment (such as central venous catheter complications) during long-term treatment. In addition, both the intravenous and enteral routes face similar challenges, namely the effects of partial clearance mechanisms of the body (e.g., mononuclear phagocytes of the liver and spleen or the first chemical modification of the liver) on the filtration or metabolism of active pharmaceutical ingredients and nonselective distribution of drugs $[10,14]$.

For example, the treatment of chronic lung infections is currently administered intravenously or orally. Although it has a good anti-infection effect, the systemic administration of

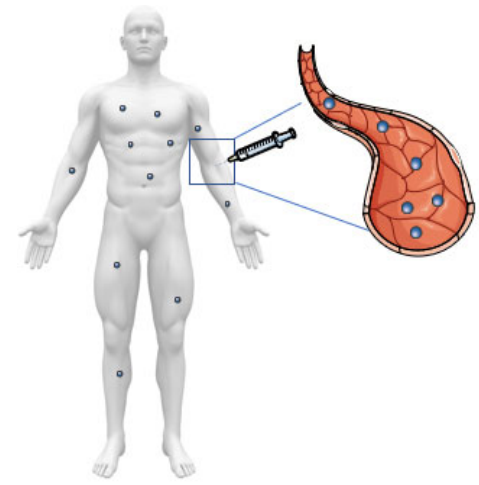

Intravenous administration

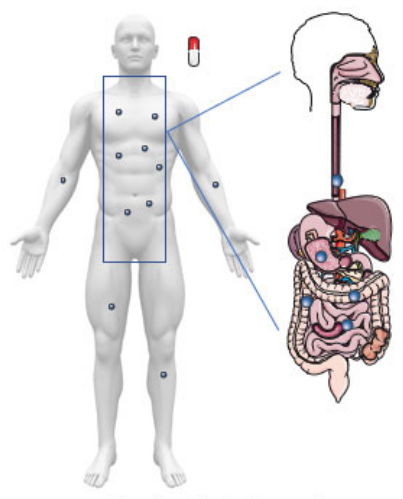

Oral administration

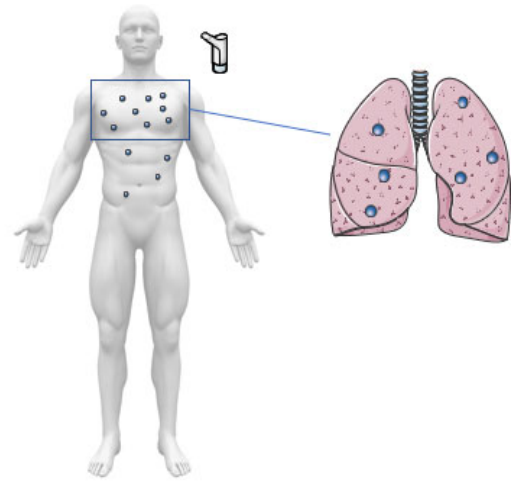

Inhalation administration

Figure 1 Different forms of administration for lung diseases. 
antibiotics has several disadvantages, such as the toxic side effects and drug resistance of repeated use of high-dose antibiotic drugs, which requires us to further improve the method of administration. Inhalation therapy has been widely considered. This method can achieve local administration, avoid adverse effects on healthy tissues, and may be the most effective strategy for treating infectious diseases of the lungs. In addition, drug delivery systems (DDSs; such as NP delivery systems) can overcome the biological barriers of the lungs and can increase the bioavailability of anti-infective drugs. The above strategies have significantly broad prospects for eliminating bacterial infections and preventing bacterial resistance. To a certain extent, the inhalation route of administration can replace intravenous administration, which is an attractive strategy. It can help in the inhalation of peptides, proteins, ribonucleic acid (RNA), deoxyribonucleic acid (DNA), and other therapeutic biological macromolecules. In addition, when compared to other drug delivery strategies, pulmonary delivery can significantly increase the bioavailability of therapeutic macromolecules [15]. Nebulized inhalation can avoid oral and intravenous high and frequent antibiotic doses; thus, antibiotics can quickly reach an effective concentration in the lesion, thereby reducing systemic adverse reactions [10].

Although inhalation therapy has several advantages, it also faces certain shortcomings. For example, the drug will be quickly cleared by alveolar macrophages, resulting in a limited time for the drug to act and a reduced bacteriostatic concentration $[15,16]$. The inhalation-based administration may be accompanied by the loss of active compounds and/or deformation of particles, which may result in difficulty in achieving a precise dosage of the active ingredient in the formulation [17]. For example, in infectious diseases, when compared to systemic drug delivery, topical administration (such as inhalation aerosol) has the following primary advantages: (1) The concentration of the drug in the lesion will increase, thereby improving the bactericidal effect and reducing the development of drug resistance. (2) There is a reduction in unnecessary drug exposure in healthy areas, thereby reducing adverse drug reactions [18-20]. According to reports, for lung infections, inhalation therapy may be the most effective treatment because antibiotics can be efficiently delivered to the lungs $[20,21]$. Inhaling antibiotics can reduce the density of bacteria in the lungs and airways and restore lung function [22]. However, inhaled antibiotics currently do not maximize the effectiveness of anti-infective treatment because it also faces the following limitations and challenges: (1) The water solubility of the drug is significantly important, and improving its water solubility helps to improve the bioavailability of the drug [23]. (2) Maintaining the antibiotic drug concentration above the minimum inhibitory concentration (MIC) is critical for bacteriostasis [24]. Overcoming the lung barrier can improve the role of drugs $[25,26]$. Besides, if antibiotics are used in large amounts without controlled release or the target disease site is not properly determined, healthy tissues can be damaged $[27,28]$. (3) Although inhaled antibiotic preparations can stop the transmission of pathogens and reduce the destruction of airway tissue, they cannot eliminate the infection [29].

The human lung tissues have alveolar regions with a large surface area. Pulmonary drug delivery can be an attractive strategy. Pulmonary drug delivery strategies demonstrate considerable prospects for improving drug therapy, whether systemic or the local use of drug therapy. Direct lung delivery may be the most ideal route for the treatment of pneumonia, lung cancer, COPD, asthma, and so on.

For example, diseases with severe lung infections such as
COPD and pneumonia are becoming increasingly difficult to treat. This may be related to the poor solubility of certain antibacterial drugs, lack of selective penetration into the diseased tissue, poor pharmacokinetic (PK) properties, and emergence of multidrug-resistant bacteria [30, 31].

The ideal route of administration must deliver as much drug as possible to the lesion. The choice of administration route depends on the chemical and physical properties, pharmacology, and toxicology of the drug and delivery vehicle, as well as the anatomical features caused by the disease. For example, in the treatment of lung cancer, COPD, tuberculosis, asthma, and so on, direct pulmonary administration may be the ideal route [32]. Pulmonary atomization is the most common local treatment method for direct pulmonary administration. It is primarily used for local lesions in the bronchi, such as in COPD and asthma. The drug is delivered directly to the lesion, and then the dynamic contour of the diseased tissue is improved to achieve therapeutic effects. Therefore, pulmonary atomization can directly deliver the drug to the diseased area and avoid the damage to other tissues and organs that is caused by the drug [33].

Lung-targeted drug delivery is a promising therapeutic strategy. This is because of the unique anatomical structure of the lungs, such as the low thickness of the epithelial barrier, large surface area of the alveolar region, high degree of vascularization, relatively low proteolytic activity, and lack of preliminary metabolism [34-36]. Certain studies have demonstrated the advantages of lung-targeted drug delivery strategies for lung diseases; moreover, these strategies can improve the therapeutic effect. Further, pulmonary administration is easy to achieve because the drug can easily reach the lung lesion through intravenous and intratracheal routes [37].

Considering that the human respiratory tract has an efficient clearance mechanism, the drugs are cleared without direct drug action in the lungs after direct lung administration. Therefore, the conventional two to four times a day dosing regimen still requires an improvement. Perhaps the treatment method of prolonging the action time of the drug may be more effective in treating certain lung diseases [38]. Recent studies have confirmed that the therapeutic effect of lung diseases can be enhanced through sustained drug action $[39,40]$. In respiratory diseases, continuous drug delivery has several advantages. For example, it can prolong the action time, improve the treatment effect, and reduce the adverse drug reactions [41, 42].

In recent years, with the progress of science and technology, nanotechnology has provided a feasible platform for improving the diagnosis and treatment of diseases. For example, when compared to the traditional drug delivery methods, nanocarriers have the advantages of prolonged blood circulation, high drug loading, low cytotoxicity, and limited immunogenicity [43-51]. One promising method to improve these key obstacles in traditional therapy is to develop engineered nanoparticles (NPs). NPs mainly classified by chemical properties such as organic NPs and inorganic NPs (Fig. 2). NPs can be used as delivery vehicles for therapeutic substances such as drugs and antibodies [30, 52].

A successful DDS must solve some of the deficiencies of existing drugs, such as (1) side effects, especially toxic ones, (2) difficulty experienced by the drug molecules in reaching the lung environment, and (3) poor water solubility [53, 54]. In recent years, more and more researchers have applied nanotechnology to drug delivery [55]. Owing to its characteristics, nano-delivery systems can help drugs overcome the disadvantage of poor water solubility and improve the biodistribution of drugs. Further, they can protect certain active pharmaceutical ingredients from being degraded by certain enzymes in the 


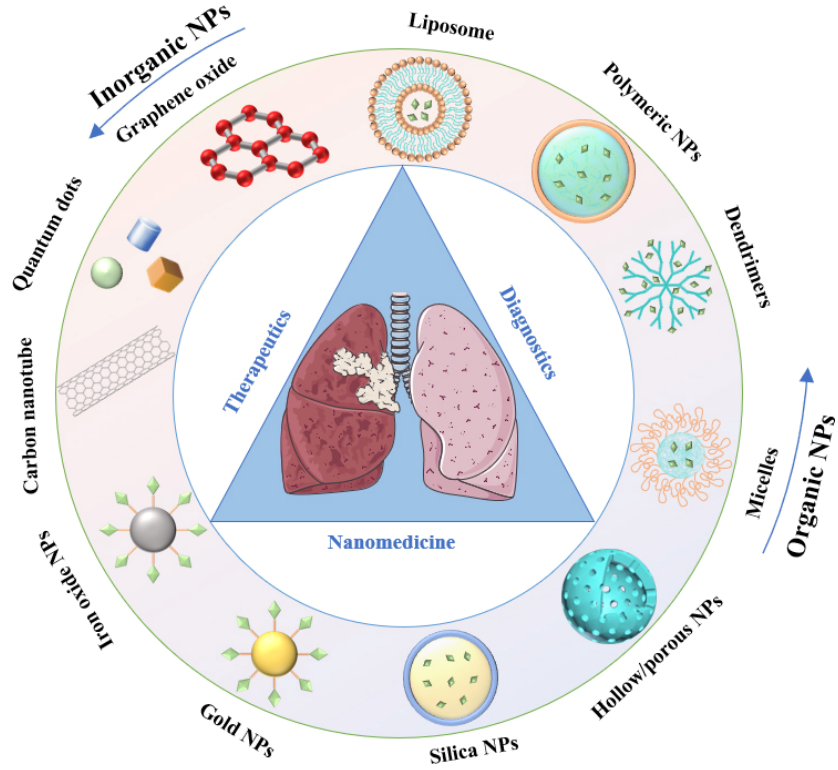

Figure 2 Schematic representation of different nanomedicine approaches in lung diseases.

body and minimize adverse drug reactions. In addition, the nano-DDS can help extend the half-life of the drug, control the release of the drug, overcome the lung barrier, and increase the permeability of the drug in the lung [56-62]. For example, for the delivery of antibiotics, NPs can maintain the drug concentration above the MIC value in a superior manner. In addition, the use of excipients on nanomaterials can also increase its affinity with the bacterial cell envelope [24]. The nano-DDS can help overcome lung obstacles (biofilm and/or mucus) and improve drug delivery efficiency, deliver the drug to the lesion more directly, improve the treatment effect, and reduce drug resistance $[63,64]$.

This article reviews the latest developments in nanotechnology pertaining to the diagnosis and treatment of lung diseases in recent years. The application value of nanotechnology was introduced from different lung diseases.

\section{Advances in nanotechnology pertaining to the diagnosis and treatment of lung diseases}

\subsection{Lung cancer}

Lung cancer has a high mortality rate, accounting for $3 \%$ of the total deaths worldwide and $19 \%$ of cancer-related deaths. In addition, its five-year survival rate is approximately $19 \%$, and the prognosis is poor. The above situation shows that the current treatment of lung cancer must be improved [65].

\subsubsection{Nanomedicine and anticancer drugs}

Doxil (Caelyx) is doxorubicin (DOX) PEGylated liposome nanodrug and it has been approved by the U.S. Food and Drug Administration (FDA) for clinical use [66]. Doxil has been used as a therapeutic drug in non-small cell lung cancer (NSCLC) and small cell lung cancer (SCLC). In phase I clinical trials, Doxil showed a single effect in metastatic or locally advanced NSCLC [67]. In another study, researchers used a triple chemotherapy regimen (Doxil, gemcitabine, and docetaxel) to treat 20 patients with advanced NSCLC who did not receive chemotherapy [68]. In phase II of the study, Doxil, cyclophosphamide, and vincristine were used in combination to treat SCLC. Research results show that nanodrugs combined with other drugs have certain antitumor effects [69]. To more effectively deliver drugs to the lungs, certain researchers synthesized methoxy PEG-PEI-poly(l-glutamic acid) copolymer as a carrier to deliver cisplatin (CDDP) and DOX. The results of the cell experiments showed that, when compared to CDDP or DOX alone, Co NPs showed higher cytotoxicity to B16F10 cells. In the in vivo experiments, Co NPs were delivered locally to the lung, especially in the highly accumulated lung tumor tissues, which are rarely observed in normal lung tissues. In addition, when compared to the use of DOX or CDDP alone, Co NPs showed better therapeutic efficacy for metastatic lung cancer [70].

Etirinotecan pegol (NKTR-102) is another polymer conjugate of the topoisomerase I inhibitor, irinotecan. The study determined that polymer-modified irinotecan can be released slowly and continuously. When compared to irinotecan, NKTR102 has shown excellent efficacy in mouse xenograft models of lung cancer and good PK characteristic in Beagle dogs, tumor-bearing mice, and Sprague-Dawley rats [71]. Certain researchers confirmed the safety profile and maximum tolerated dose (MTD) of NKTR-102 in cancer patients. The study tested 76 cancer patients, 18 of whom were lung cancer patients (15 NSCLC and 3 SCLC). The results showed that MTD varies with the frequency of administration. If the frequency of administration is lower, higher doses can be tolerated. Antitumor effects were shown in one-third of the patients, and eight patients achieved partial responses (two of them were lung cancer patients) [72]. Phase II clinical trial of NKTR-102 for the treatment of NSCLC patients is currently underway [73]. Onivyde is a liposome nanoformulation carrying irinotecan. Onivyde has the effect of controlled release, which can reduce the toxicity of drugs while improving the efficacy [74]. A recent clinical study showed that Onivyde has excellent anticancer activity and longer drug circulation time than free drugs [74, 75]. A phase III clinical trial using Onivyde to treat patients with SCLC who have progressed after platinum-based first-line therapy is underway (NCT03088813).

Paclitaxel (PTX) is a chemotherapeutic agent for lung cancer patients. It is characterized by poor water solubility, low bioavailability, high toxicity, and the requirement for the utilization of excipients with severe side effects, which limits its use [76]. Currently, PTX nanoformulations have been approved for clinical use. It can improve the efficacy and reduce the toxicity and side effects caused by PTX. Genexol-PM is the first PTX nanoformulation approved for the treatment of NSCLC. In addition, studies have shown that after PTX is encapsulated in nanoscale polymer micelles, higher doses of PTX can be administered to patients without increasing toxicity [77]. Abraxane is a protein-based nanoformulation that has been approved for the first-line treatment of advanced NSCLC [78]. It contains PTX NPs that bind to albumin. When compared to Genexol-PM, it can administer higher doses of PTX [79]. Several studies have shown that, when compared to free PTX, PTX nanoformulations show better drug efficacy and PK characteristics. The copolymer, i.e., poly(lactide-co-glycolide) (PLGA), nano-delivery vehicle formed by glycolic and lactic acids has low toxicity and high biocompatibility. Certain researchers loaded PTX into PLGA NPs, and the nanoformulation showed good biocompatibility and enhanced the uptake of PTX in lung cancer cells. In addition, the results of the study show that PLGA-PTX NPs can significantly increase apoptosis and reduce the volume of tumorspheres derived from $\mathrm{LL} / 2$ and A549 cells. Biodistribution studies have shown that PLGA-PTX NP drug circulation time is increased when compared to free PTX; moreover, the accumulation in lung and brain tissues is greater [80]. Resistance to the tyrosine kinase inhibitors targeting the epidermal growth factor receptor 
(EGFR TKIs) in NSCLC usually appears after 9-13 months of medication. Studies have shown that the combined use of EGFR TKI and other chemotherapy drugs can help overcome the resistance of EGFR TKI. Certain researchers developed an encoded afatinib (AFT) and PTX lung microsphere system for the treatment of NSCLC resistant to EGFR TKIs. The results of the study showed that AFT and PTX have a synergistic effect and show excellent therapeutic effects in drug-resistant NSCLC cells. In vivo experiments have shown that PTX and AFT can maintain high concentrations in the lungs for $96 \mathrm{~h}$. This drug delivery strategy provides research directions for EGFR TKI-resistant lung cancer [81].

CDDP can bind with DNA and cause cross linking, which can destroy the function of DNA and inhibit cell replication $[82,83]$. CDDP is widely used in cancer treatment because of its good antitumor activity and broad-spectrum anticancer activity. However, drug resistance to CDDP caused by long-term administration or spontaneous mutation of tumor cells results in it losing its physiological activity in the course of treatment, which is still one of the primary reasons for the failure of clinical treatments.

According to the mechanism of CDDP resistance in lung cancer cells, certain researchers proposed a new idea to reverse the drug resistance. They copolymerized carboxymethyl chitosan and diallyl disulfide to obtain nanogel and further graft valproic acid, giving the ability of reactivation to CDDP and promoting early apoptosis. Subsequently, CDDP-tolerant lung cancer cells (A549/DDP) were used as models to study the anticancer activity of the nanogel. The results showed that the vector could reverse CDDP resistance using the following mechanisms: (1) reactivating CDDP through valproic acid-induced high-level G2/M block (up to 3.2 times higher when compared to the control group); (2) promoting early apoptosis of tumor cells by enhancing reactive oxygen species (ROS)-p53 crosstalk, further inhibiting CDDP resistance. The results of in vivo experiments showed that CDDP was well tolerated in A549/DDP mice model; moreover, the tumor volume was not inhibited by CDDP, and the tumor volume increased rapidly in the experimental cycle. However, in the nanoscale gel loaded with CDDP through the tail vein, the tumor-suppressing effect was obvious, and the survival time of A549/DDP in mice was significantly prolonged. These results provide a new idea for clinical treatment for reversing CDDP resistance and drug-resistant tumors. The detailed process is illustrated in Fig. 3 [84].

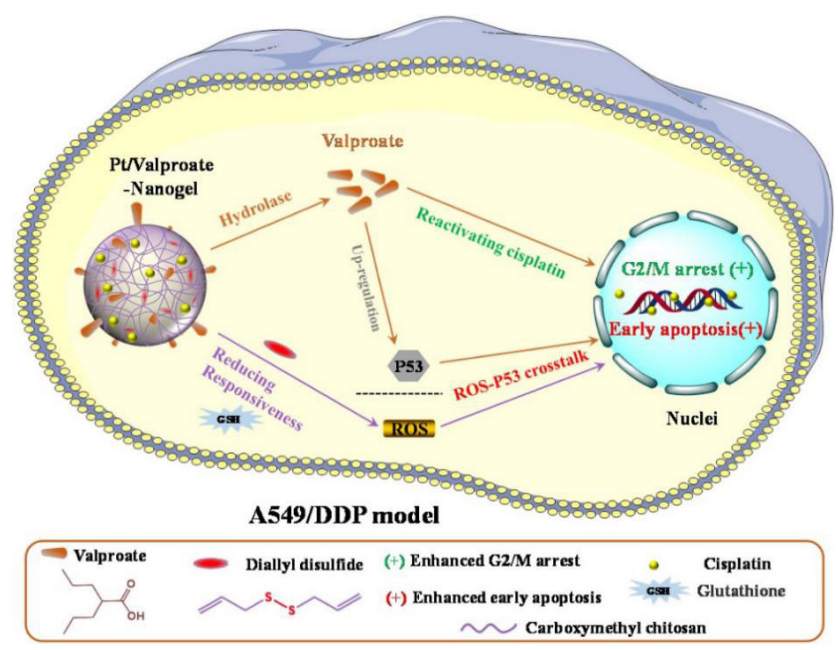

Figure 3 Model showing how Pt/valproate-D-nanogel reactivates cisplatin and enhances early apoptosis in A549/DDP Cell. Reproduced with permission from Ref. [84], ( Elsevier Ltd, 2020.
Researchers developed a glutathione (GSH)-responsive polyurethane NP that is sensitive to GSH in lung cancer cells. GSH-sensitive NPs loaded with CDDP can release CDDP under the premise of dose-dependent GSH. In addition, when compared to the same concentration of free CDDP, the survival rate of NP-treated A549 cells was significantly reduced in vitro. Moreover, the study also showed that NPs have a higher biodistribution in the tumor area and a stronger tumorsuppressing effect [85]. Lipoplatin is a liposome nanoformulation of CDDP. When compared to the traditional CDDP, lipoplatin has shown a strong anticancer effect in clinical trials [86]. In addition, a new clinical study showed that gemcitabine combined with lipoplatin is a good treatment option for advanced NSCLC [87].

\subsubsection{Nanomedicine and lung cancer immunotherapy}

AFT has a good therapeutic effect on NSCLC. Certain researchers prepared near-infrared persistent luminescence nanomaterials (NIR PLNs) and used it as a drug delivery carrier for AFT. In addition, the NIR PLNs can also track NSCLC cells. The NIR PLNs can effectively load targeted drugs (such as AFT); moreover, AFT nanoformulations (i.e., AFT-PLN) show unique anticancer therapeutic benefits. In addition, after modifying the PLN (AFT-PLN@MAp) with the specific targeting aptamer melanoma-associated antigen 3 protein (MAp), the nanoformulations showed stronger targeting ability [88].

Immunotherapy has become an effective treatment strategy against lung cancer. Human programmed death-ligand 1 (PDL1), in addition to being an important checkpoint gene in the immune system, is also crucial for the survival of cancer cells through its inherent signaling activity. Studies have shown that drugs targeting programmed cell death protein 1 (PD-1) and its ligand PD-L1 can effectively prolong the survival of patients with advanced NSCLC $[89,90]$. Owing to the special characteristics of gold NPs (GNPs), their potential applications in tumor imaging and therapy have been extensively studied. A new nanoplatform GNPs@PSS/PDADMAC-siRNA was designed and fabricated by sequentially coating the GNPs with poly(-diallyldimethylammonium chloride) (PDADMAC) and poly(sodium 4-styrenesulfonate) (PSS) to carry small interfering RNA (siRNA). The prepared nanoplatform can not only down-regulate the expression of PD-L1 but can also be used as a photothermal agent to perform a therapeutic role in lung cancer [91].

\subsubsection{Active targeting of nanomedicines for lung cancer}

Designing targeted delivery systems with both imaging and therapeutic functions is significantly popular in the field of nanomedicine. Certain researchers developed PEGylated AgS quantum dots (QDs) with the function of cetuximab (Cet) antibodies and containing the anticancer drug 5-fluorouracil (5FU). Cet-conjugated QDs can effectively and selectively deliver 5FU to A549 cells, and significantly increase cell death associated with apoptosis. The results also show that the therapeutic effect of 5FU delivered to A549 cells through Cet-coupled AgS QDs is improved, which is a synergistic result of enhancing receptor-mediated uptake of NPs. Moreover, without adding autophagy inhibitors, drugs can also inhibit autophagy [92].

Approximately $50 \%$ of lung cancer patients overexpress the EGFR; moreover, Polo-like kinase1 (PLK1) is a key regulator of cell mitosis. Inhibiting the PLK1 expression can enhance radiation sensitivity. For NSCLC, certain scholars developed a radiosensitizer and nanotargeted therapy. The researchers coupled the NPs to Cet to target EGFR and load siRNA that 
can inhibit the expression of PLK1. Research results showed that the nanoformulation (C-siPLK1-NP) can effectively target EGFR+ NSCLC cells, down-regulate the expression of PLK1, and thereby inhibit cell proliferation. The synergistic use of radiosensitizers and C-siPLK1-NP can enhance antitumor ability. In addition, the treatment results of C-siPLK1-NP in an orthotopic lung tumor model show that the application of C-siPLK1-NP can effectively inhibit tumor growth and prolong survival time [93].

Active targeting is an effective treatment strategy that maximizes the amount of drug components that reach the target tissue, thereby improving treatment efficiency and minimizing adverse reactions [94, 95]. BIND-014 is a PTX polymer nanoformulation that can target prostate-specific membrane antigen [96, 97]. When compared to nontargeted docetaxel NPs and solvent-based docetaxel, BIND-014 showed a greater reduction in average tumor weight in the NSCLC mouse xenograft model. In addition, in vivo experiments showed that BIND-014 improved PK characteristics when compared to solvent-based docetaxel [97]. In a phase I clinical trial, the efficacy of BIND-014 was studied in patients with metastatic or advanced tumors. According to the conclusion of the study, it was determined that the nano-delivery strategy can prolong the release time of docetaxel in plasma [98]. For lung cancer patients whose previous treatment with platinum-containing drugs were failures, two phase II clinical trials evaluated the efficacy of BIND-014 as a second-line treatment for lung cancer (NCT01792479; NCT02283320). In recent years, therapeutic agents such as DNA, microRNA (miRNA), and siRNA have shown promising prospects for treating lung cancer and studies have evaluated their therapeutic efficacy [99]. TargomiR is a type of NP targeting specific antibodies against EGFR, which carries a mimic miRNA based on miR-16 [100]. Research results show that TargomiR demonstrates safety and early antitumor activity [101].

Certain researchers developed coated cationic lipid NPs embedded in miR-660 (CCL660). In a lung cancer-based mouse model, the results showed that miR-660 expression was downregulated, and its substitute could inhibit the mouse double minute 2 homolog/p53 axis and inhibit the growth of lung cancer. In addition, in two different p53 wild-type subcutaneously metastasized lung cancer patient-derived xenografts (PDXs), systemic delivery of CCL660 increased the miRNA level in the tumor, reduced the proliferation of cancer cells, significantly reduced tumor growth, and did not produce off-target effects. Interestingly, the antitumor effect of CCL660 in p53 mutant PDX was also observed, and miR-660 also had an inhibitory effect on lung cancer metastasis. In addition, the results showed that the synthesized miR-660 lipid NPs have no immune off-target or acute/chronic toxicity [102].

Oncoplex is a type of nontargeted nanovesicle preparation, which encapsulates the tumor suppressor 2 gene, TUSC2. The TUSC2 gene can express tumor suppressor 2 protein in NSCLCs [103]. In patients with metastatic lung cancer after previous platinum-based chemotherapy (NCT00059605), a phase I clinical study was conducted to evaluate the efficacy of oncoplex in the treatment of lung cancer. The results show that oncoplex is safe and effective for patients with lung cancer. In recent years, the development of nanovaccines against lung cancer has shown great prospects. Researchers used antiMUC1 as a candidate peptide to prepare nanovaccines. MUC1 is a glycoprotein antigen of the lung cancer cell membrane. L-BLP25 (stimuvax) is a kind of liposome nanovaccine targeting the MUC1 antigen [104]. In a previous clinical study, L-BLP25 showed significant survival benefits in patients with stage III NSCLC [105]. The therapeutic effect of L-BLP25 on lung cancer was also demonstrated in other clinical trials (NCT01015443) [106].

\section{$2.2 \mathrm{CF}$}

$\mathrm{CF}$ is an autosomal recessive disease that can cause respiratory tract infections, lung obstruction, and even respiratory failure due to excessive mucus production. The current primary treatment options are anti-infection treatment, bronchodilator, and gene therapy [107]. Because CF is caused by gene mutation of CF transmembrane conductance regulator (CFTR), gene therapy has broad prospects. Currently, gene therapy primarily uses nonviral or viral vectors to transfer the CFTR gene to target sites for treatment [107]. It is worth noting that nonviral vectors (such as NPs) have more advantages than viral vectors, such as lower production difficulty and cost, longer shelf life, lower immune regulatory response, and better drug tolerance [108].

A clinical study used plasmid DNA to carry the gene encoding CFTR and encapsulated it in PEG NPs. The research results show that this method has good safety and high genetransfer efficiency, and nano preparations can be used as powerful candidates for gene-transfer vectors [109]. Another study conducted a preclinical evaluation of a variety of nonviral vectors. The results show that cationic liposome preparation GL67A is the most effective delivery vehicle [110-112]. Phases I and IIa of the study showed that using liposome NPs (GL67A) to deliver aerosolized PGM169 plasmid DNA encoding the CFTR gene is a good choice and has excellent therapeutic effects. In the clinical trial in phase IIB, the clinical benefits of PGM169/GL67A in patients with CF were evaluated. The results showed that the aerosol preparation treated with PGM169/GL67A was well tolerated and continued to improve lung function parameters [113]. In the clinical trial of phase IIb, the PGM169/GL67A was administered once a month for one year through a sprayer. The results showed that FEV1 was significantly increased and lung function was improved, and the PGM169/GL67A preparation was safe (NCT00789867) [114].

Although several researchers have been trying to correct the potential genetic defects of CFTR for many years, the treatment of CF with gene preparations has not yet been fully translated into clinical applications. In recent years, RNA therapy mediated by NPs is a promising technology. Robinson et al. used lipidbased NP (LNP) to package and deliver chemically modified CFTR mRNA (cmCFTR) to treat CF to restore chloride secretion. Their results confirmed that chemically modified mRNA (cmRNA) could successfully express the desired transcripts. Moreover, the mechanism and efficacy of LNP-cmRNA is comparable to that shown by ivacaftor [115]. Certain researchers prepared chitosan-coated PLGA NPs and delivered human CFTR (hCFTR) encoded by cmRNA to the lungs of CFTRdeficient mice by intratracheal and intravenous administration. In the study, the researchers determined that key lung function parameters were restored in general (FEV1 increased significantly) and chloride secretion was reduced [116]. Recently, researchers developed a LNP for delivering cmCFTR to the bronchial epithelial cells of patients, which increases the membrane-located CFTR, which can improve its use as a chloride channel. In addition, in vivo experiments showed that, in CFTR knockout mice, the use of LNP-cmCFTR in the nasal cavity for at least 14 days resulted in the restoration of CFTRmediated secretion of chloride to conductive airway epithelial cells. In addition, on the third day after transfection, by observation, CFTR had the highest activity. The above treatment 
response is comparable to the curative effect of the currently approved drug ivacaftor. It can be observed that mRNA therapy mediated by NPs has high clinical application value [115]. Tagalakis et al. developed a receptor-targeted nanocomplex (RTN) for the treatment of CF. RTN is prepared by combining targeted peptides, liposomes, and siRNA. The results showed that siRNA transfection mediated by NPs could repair mucociliary defects in vivo [117].

A previous study introduced a new nonviral vector based on cell-penetrating peptides, which utilizes glycosaminoglycanbinding enhanced transduction (GET) for efficient gene transfer. To adapt the GET peptide for effective in vivo delivery, researchers modified it with PEG to stabilize the particles and maintain gene-transfer activity. The results of the multiparticle tracking technology show that the PEG-GET complex can navigate the mucus network and rapidly diffuse through the sputum samples of CF patients. When tested in a live mouse lung model, the PEGylated particles showed better biodistribution, better safety, and higher gene-transfer efficiency than non-PEGylated complexes. In addition, the researchers also compared it with PEI NPs, and the gene expression of PEGylated particles was significantly enhanced. Summarizing the above results, combining the novel GET peptide for enhanced transfection with an adjustable PEG coating will be a development in gene therapy for pulmonary CF [118]. Pulmonary administration of drugs in CF still faces several difficulties because viscoelastic mucus can prevent particles and drugs from penetrating the mucus barrier. Scholars have used combined peptide presentation phage libraries and next-generation sequencing to identify hydrophilic, net-neutral charged peptide coatings, which have a mucus penetration that is approximately 600 times higher than the control and are not wrapped or polymerized. When compared to the PEGylated NPs, the uptake efficiency of lung epithelial cells is further improved, airway retention time is extended, and biodistribution is further improved. The peptide coating mentioned in this study effectively overcomes the delivery barrier and performs better than standard PEG surface chemicals, efficiently achieving mucus penetration, and solving some of the challenges currently faced by PEG and other chemicals. This strategy is expected to improve the therapeutic effect of CF and other diseases [119].

CF is a genetic disease caused by CFTR mutations. CFTR is a type of chloride channel that exists on the surface of epithelial cells. Recently, certain scholars proposed the idea of combining lumacaftor and ivacaftor to treat CF. Lumacaftor can increase the number of CFTR proteins localized on the cell surface, while ivacaftor can increase the probability of CFTR channel opening. In addition, the therapeutic effect of drugs can be improved by inhalation. They chose a nanostructured lipid carrier (NLC) for direct inhalation and conducted drug-loading tests in vitro and in vivo. The results showed that NLC has high drug-loading capacity and can be internalized into target cells. In addition, the prepared NPs can help restore the normal expression of CFTR protein [120]. Currently, two types of liposome antibiotic preparations have been developed, including liposome AM and liposome ciprofloxacin. Liposome AM was approved by FDA for clinical application in 2018. Phase II clinical trial of liposome ciprofloxacin has been completed. The experimental results show that the efficacy of liposome ciprofloxacin is better than that of nonencapsulated ciprofloxacin [121, 122]. Researchers have successfully encapsulated ciprofloxacin in PLGA NPs, and the prepared nanocomposite has good anti-protective antigen strain efficacy and considerable mucus penetration ability. Moreover, the nanocomposite has a higher drug-loading capacity. In addition, it was reported that ciprofloxacin NPs have enhanced antibacterial activity, indicating that nanomaterials have broad prospects as delivery carriers of CF antibacterial drugs [123]. Tobramycin was encapsulated in PEG-coated PLGA NPs and used to treat CF and the nanodrugs had enhanced antibacterial activity when compared to free drugs; further, they had better permeability in mucus and bacterial biofilms [124].

\subsection{COPD}

Chronic respiratory disease is a common illness and includes multiple diseases [125]. According to the data statistics of the World Health Organization (WHO), the lower respiratory tract infection represented by COPD has become the third leading cause of death worldwide, with nearly 4 million people dying each year, and the number of deaths is increasing annually [126]. Nowadays, the worldwide focus is on methods to effectively prevent and control chronic respiratory diseases.

The lack of effective drugs is the primary reason for the difficult in curing chronic respiratory diseases, especially COPD. There are several types of drugs in the clinical treatment of COPD, such as antibiotics, bronchodilators, and glucocorticoids. Although they have certain therapeutic effects, they are not specific drugs. Long-term use of drugs can easily cause serious side effects on patients. In addition, another important reason why COPD is not easy to cure is that conventional treatment drugs are difficult to deliver effectively, and the drug delivery efficiency is low. COPD often results in abnormal pulmonary inflammation, which leads to cough and secretion of mucus in the airway, which forms a "barrier" that blocks the effective delivery of drugs $[57,127,128]$. At the same time, a large number of bacteria growing in mucus are gradually colonized, forming a dense bacterial biofilm that cannot be eliminated by drugs and results in drug resistance [129-131].

Amikacin (AM) is a commonly used antibacterial drug for the treatment of COPD combined with gram-negative bacilli lung infections $[132,133]$. Previous studies have shown that AM delivered by NPs can increase the drug concentration in lungs and reduce systemic adverse reactions [134, 135]. Because of this treatment dilemma, the research team used ion crosslinking method to combine chitosan with black phosphorus QDs, and through PEG surface modification, successfully constructed a nanodrug controlled-release carrier mediated by black phosphorus QDs (PEG@CS/BPQDs-AM NPs), which can penetrate the pulmonary mucus barrier, enhance the drug delivery ability, and demonstrate a synergistic effect on COPD. The results showed that the hydrophilic PEG and positively charged chitosan could help the nanocarrier penetrate the mucus layer and adhere to epithelial cells. Then, the black phosphorus QDs in the nanocarrier could be rapidly oxidized and degraded to produce phosphate ions, which can cleave the nanospheres to promote drug release. Simultaneously, the oxidative degradation of black phosphorus QDs also promoted the protonation of amino groups in chitosan, enhanced the antibacterial effect of chitosan, and effectively avoided the formation of a biofilm. Animal experiments showed that PEG@CS/BPQDs-AM NPs can significantly improve the symptoms of COPD airway obstruction in mice, enhance the inhibitory effect of PA, and significantly reduce the toxicity and side effects of treatment. This study provides a new treatment strategy to solve the problem of poor drug treatment effect caused by the mucus barrier of respiratory diseases. The detailed process is illustrated in Fig. 4 and the results of mucus-penetration and antibacterial performances of various samples are showed in Fig. 5 [136].

ROS are closely related to the occurrence and development of COPD. The excessive production of ROS can lead to a 


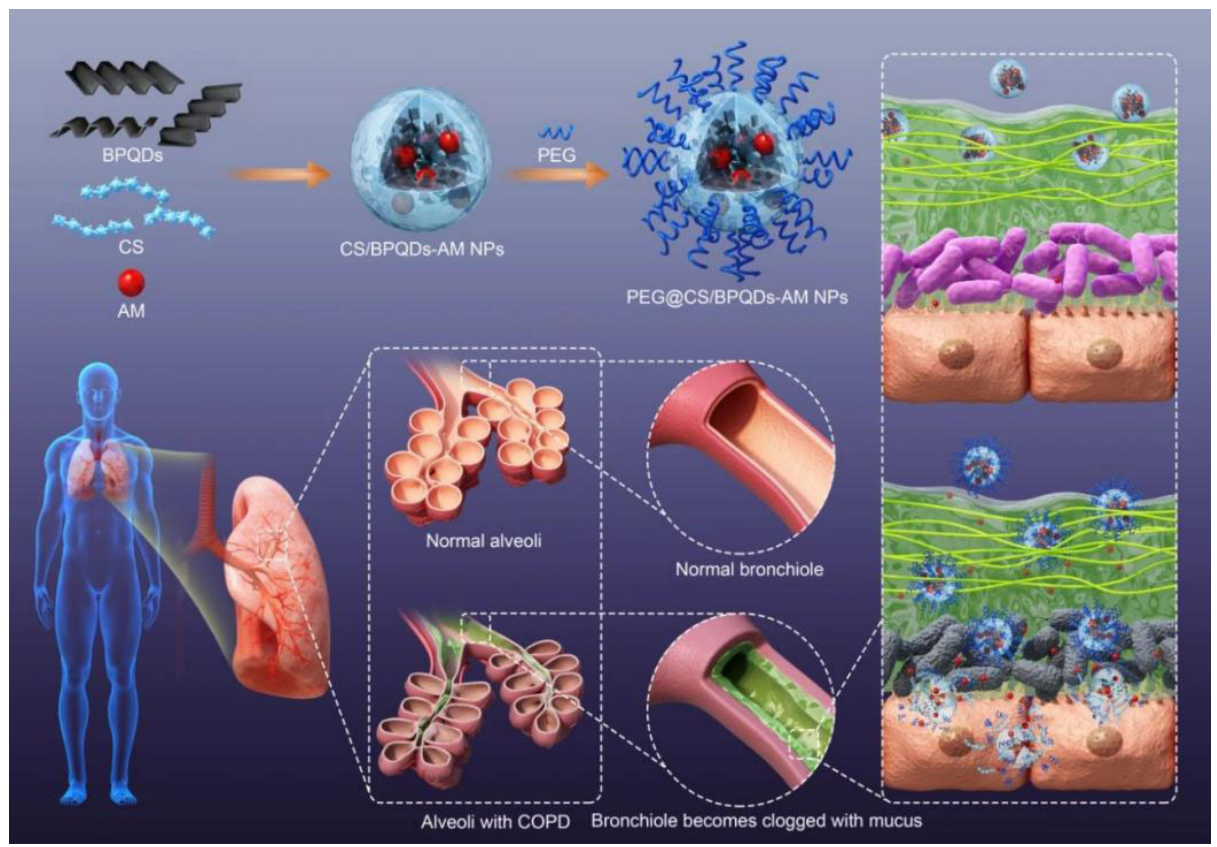

Figure 4 Schematic diagram illustrating the fabrication process of PEG@CS/BPQDs-AM NPs and biomedical application as a drug delivery system that penetrates pulmonary mucus layer, releases therapeutic agents, and inactivates pathogenic bacteria to alleviate COPD. Reproduced with permission from Ref. [136], ( $)$ Wiley-VCH GmbH, 2020.

decrease in histone deacetylase 2 (HDAC2), which leads to glucocorticoid resistance. Therefore, identifying ways to reduce ROS levels and glucocorticoid resistance will help us improve the prognosis of COPD. To achieve this goal, researchers have developed core-shell lipid-polymer NPs (LPNs), which are composed of polylactic acid coated with an effective antioxidant $\mathrm{Mn}$ porphyrin dimer (MnPD). Its core is composed of cationic lipid (1, 2-dioleoyl-3-trimethylammonium-propane (DOTAP)) shell and HDAC2-encoding plasmid DNA (pHDAC2). The expression level of HDAC2 was significantly increased by the transfection of pHDAC2 and clearance of ROS by MnPD. In addition, the results showed that after LPN therapy, the expression level of interleukin-8 decreased, indicating that LPN therapy can effectively improve the hormone resistance of the COPD model. Based on the synergistic effect of HDAC2 expression and ROS clearance, this strategy provides a new direction for improving glucocorticoid resistance in COPD patients [137].

In recent years, miRNAs have been increasingly studied for the treatment of COPD. Recently, researchers prepared NPs containing cationic lipid DOTAP for delivery of miR-146a to reduce the expression of interleukin-1 receptor associated kinase-1 (IRAK1) gene in COPD patients. The study determined that the prepared NPs can effectively aggregate in lung cancer cells and can achieve sustained release. Moreover, miR-146a as miR-146a-NPs can reduce the expression of target gene IRAK1 to $40 \%$ at a certain concentration. The above results show that poly(glycerol adipate-co- $\omega$-pentadecalactone) NPs deliver broad prospects for miR-146a treatment of COPD [138].

Several researchers have researched the delivery of selective drugs and genes based on PLGA nanosystems to treat COPD $[139,140]$. Certain research results show that PLGA NPs have some disadvantages. For example, when using emulsifier polyvinyl alcohol to prepare NPs, the surface of NPs can be highly negatively charged $[45,141,142]$. In experimental animals, PLGA NPs were quickly removed by the airway defense system. To improve the properties of PLGA NPs, researchers covered the surface of PLGA NPs with PEG, which not only has the function of airway defense, but also has the characteristics of escaping mucus. PLGA PEGylation can also increase the retention time of NPs, thus enhancing the accumulation tendency of NPs in target cells and reducing the molecular load of NPs. In addition, NPs mediated drug or gene delivery has the additional advantage of targeting specific cells in the body $[45,141,142]$.

Early diagnosis of COPD can significantly improve the prognosis. Currently, the gold standard for the diagnosis and monitoring of COPD is a vital capacity measurement [143]. Moreover, in the early stages of COPD, conventional spirometry could not accurately reflect the true condition of the disease because no obvious respiratory symptoms occurred [144]. Studies demonstrated that an electronic nose based on nanosensors can be considered as a method to diagnose COPD, and can be used to identify asthma, COPD, and lung cancer $[145,146]$. A clinical trial (NCT01976117) showed that the electronic nosebased method can also be used to analyze the colonization of pathogens in patients with COPD. The electronic nose is a convenient, noninvasive, and reliable detection method, which can be used to analyze bacterial colonization in patients with COPD [147].

\subsection{Pulmonary tuberculosis}

The current treatment options for tuberculosis are relatively mature; however, antituberculosis drugs still have certain shortcomings. For example, the medication cycle is long and there are several side effects. The nano-DDS may further improve the efficacy of the antituberculosis drugs [148-150]. In recent years, several nanotechnologies for the diagnosis and treatment of tuberculosis have been thoroughly researched and have achieved good results [151-153].

The requirement for long-term daily administration is a major challenge in current antituberculosis treatments, which can easily lead to poor patient compliance. Because tuberculosis is highly contagious, if it is not treated formally, it may not only cause health problems in patients, but also pose risks to public health. Therefore, improving the treatment compliance of the patient is also our goal. In addition, the drugs currently used for antituberculosis also have different degrees of adverse 


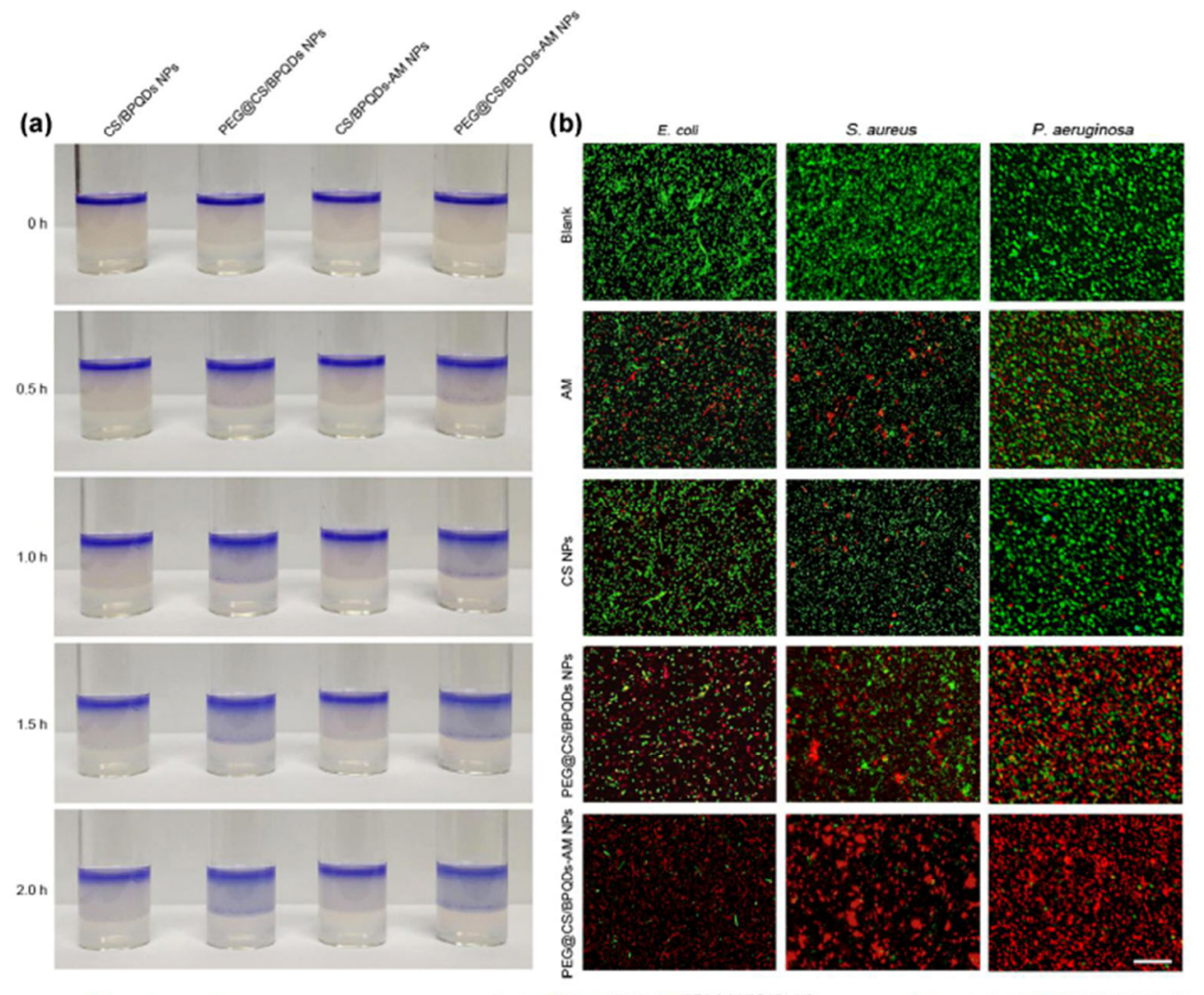

(c) $A M=C S N P S=P E G G C S I B P C O S N P S$

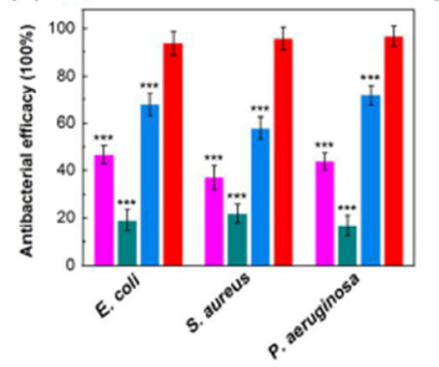

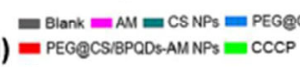

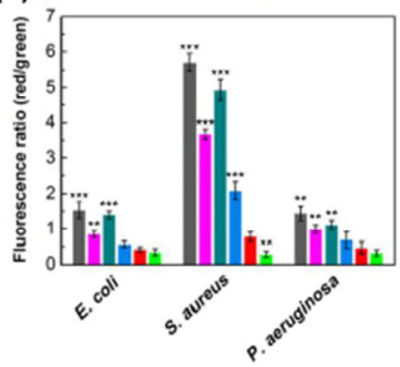

(e) $=$ PEGgCSIBPCDS.AMNPS

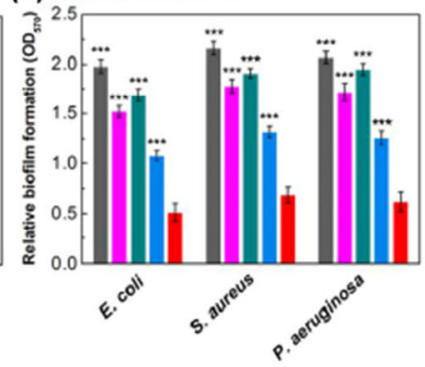

Figure 5 Mucus-penetration and antibacterial performances of various samples. (a) Visual inspection of various samples penetrate through the artificial mucus layers. (b) Live/dead staining of E. coli, S. aureus, and P. aeruginosa bacterial strains treated with various samples for $12 \mathrm{~h}$ (live cell are stained green and the dead ones are stained red, scale bar $=50 \mu \mathrm{m}$ ). (c) Antibacterial rates of various samples after the incubation with different bacterial suspensions $(5 \mathrm{~mL}, 106 \mathrm{CFU} / \mathrm{mL}$ ) for $12 \mathrm{~h}$. (d) Red/green fluorescence staining of bacterial membrane potential. (e) Relative biofilm formation after the different treatment for $12 \mathrm{~h}$. Reproduced with permission from Ref. [136], (c) Wiley-VCH GmbH, 2020.

reactions, such as short half-life, increase in liver and kidney toxicity, and rapid clearance from the body [154].

Researchers have developed multimetallic particles (MMP) containing $\mathrm{ZnO}$ NPs and Ag NPs that are biodegradable and can be applied to deliver the antituberculosis therapeutic agent to Mtb-infected macrophages. The in vitro macrophage infection model proves that Mtb-infected THP1 cells demonstrate a good absorption of MMP, and the release of ZnO NPs and Ag NPs in the macrophage endosomal system significantly increases the effectiveness of rifampicin. This drug delivery strategy can efficiently transport antituberculosis therapeutic agents to infected alveolar macrophages by improving the permeability of the Mtb membrane. In addition to having a therapeutic effect in drug-sensitive Mtb, the nano-DDS can also improve the therapeutic effect on drug-resistant Mtb strains [155].

As the mononuclear phagocytes engulf the Mtb, mononuclear macrophages can be used as the target location of nanodrugs. Therefore, NPs can target and efficiently deliver plasma drugs to Mtb-infected mononuclear phagocytes, thereby avoiding off-target toxicity and improving therapeutic effects. Researchers developed two different sizes (100 and $50 \mathrm{~nm})$ of stimuli- responsive mesoporous silica NPs and used them as a prodrug structural carrier for the antituberculosis drug, isoniazid. The drug passes through the formation of hydrazone bonds and is captured by aldehyde-functionalized NPs, and is coated with polyethyleneimine (PEI)-polyethylene glycol (PEG). The preparation of nanometer drugs can release the drugs spontaneously under acidic $\mathrm{pH}$ value in the body. Studies have shown that Mtb-infected mononuclear phagocytes can efficiently engulf isoniazid-loaded PEI-PEG-coated NPs, and kill the intracellular bacteria. When compared to free drugs, the NPs are not only well tolerated, but also have a better therapeutic effect [156].

Previous studies have shown that NPs made of cross-linked poly- $\beta$-cyclodextrin (p $\beta C D)$ can be applied as an ideal carrier for delivering antituberculosis drugs in the lungs. Recently, a study showed that $\mathrm{p} \beta \mathrm{CD}$ NPs have antibacterial properties in addition to being a drug delivery vehicle. The study determined that empty $p \beta C D$ NPs continued inhibiting the infection of Mtb after its administration to the lungs of mice. $p \beta C D$ can prevent the colonization of macrophages by Mtb and does not produce toxicity in the body. In addition, $\mathrm{p} \beta \mathrm{CD}$ can also induce 
macrophage apoptosis and promote the exhaustion of infected cells [157].

Certain researchers encapsulated several nanomaterials in lysosomes to promote the removal of phagosome Mtb; however, the results are not ideal and the treatments still face several challenges. A study showed that Se NPs have a bactericidal effect on Mtb. At the same time, a new type of nanomaterial-assisted antituberculosis strategy was further reported. The synergy of macrophage-targeted isoniazid-Se (Ison@Man-Se) NPs was used to induce phagocytic Mtb destruction. Studies have shown that the Ison@Man-Se NPs can enter macrophages and accumulate in lysosomes, and then release isoniazid. Moreover, Ison@Man-Se/Man-Se NPs further promoted the fusion of Mtb into lysosomes; consequently, lysosomes cooperated with isoniazid to destroy Mtb. The detailed process is illustrated in Fig. 6 [158].

It is well known that the conventional gold standard in detection methods for diagnosing tuberculosis has the problems of low sensitivity and high cost. In recent years, considering the superiority of nanotechnology, certain scholars have developed high-throughput diagnostic methods based on nanotechnology for diagnosing tuberculosis, such as nanodisk mass spectrometry. Culture filtrate protein $10 \mathrm{kDa}(\mathrm{CFP}-10)$ and $6 \mathrm{kDa}$ early secretory antigenic target (ESAT-6) are indicator biomarkers for tuberculosis [159, 160]. A nanodisk mass spectrometry assay, by coupling ESAT- 6 and CFP-10 antibodies to silica NPs, performed sensitive multiple quantitative analysis of ESAT- 6 and CFP-10 on suspected tuberculosis cases. Clinical sensitivity and specificity are required for the rapid determination of active Mtb. CFP-10 and ESAT- 6 can be quantified even at low concentrations. This test method can not only assess the severity of an active tuberculosis infection, but also the therapeutic effect [161, 162].

Certain scholars successfully developed a gold-copper nanoshell dot-blot immunoassay for the detection of CFP-10. The principle of this detection method is based on the reduction of copper ions on the GBP-CFP10G2-gold NPs conjugate (where GBP and G2 indicate the gold-binding peptide and Group 2 antibody genes, respectively). The conjugate has antigen-binding and gold-binding affinities, which help in the appearance of red spots that can be observed with the naked eye. The concentration of tuberculosis antigen CFP-10 is directly proportional to the spot intensity, and its detection limit is $7.6 \mu \mathrm{pg} / \mathrm{mL}$. The method can detect CFP-10 antigen in clinical urine samples with high detection sensitivity and specificity. This method can be used for accurate and reliable real-time detection of CFP-10 and is significantly valuable for the early diagnosis of tuberculosis [163].

\subsection{Pneumonia}

\subsubsection{Bacterial infections}

$\mathrm{AM}$ is one of the antibiotics commonly used to treat lung diseases. It can be used to treat pulmonary infectious diseases caused by drug-resistant bacteria such as PA. Currently, AM liposome NPs have been approved by FDA for clinical use. When compared to free drugs, AM liposome has better pharmacodynamics and PK characteristics [164]. The liposomal AM can continuously release and increase the stability of the drug [165]. In addition, nanopackaged AM can also be administered using an aerosol $[165,166]$. Atomization administration can maximize the efficacy of drugs and reduce the side effects [167].

Ciprofloxacin is also one of the antibiotics commonly used to treat lung infections [168]. A preclinical study showed that the atomized liposome ciprofloxacin has good PK properties. According to the results of in vitro studies of Mycobacterium avium and Mycobacterium abscessus, when compared to free ciprofloxacin, nebulized liposome ciprofloxacin has better efficacy [169]. In addition, in vivo experiments in mice showed that the half-life of aerosolized liposome ciprofloxacin was almost $8 \mathrm{~h}$ longer than that of free ciprofloxacin [170]. In terms of efficacy, when compared to the unencapsulated ciprofloxacin, liposome ciprofloxacin has stronger anti-infective capacity in mice [171]. Pulmaquin is a kind of pharmaceutical preparation that is composed of free ciprofloxacin and liposome ciprofloxacin in a certain proportion [169]. In phase II of a study, Pulmaquin showed significant antibacterial effects [168]. In two subsequent phase III studies, the efficacy of Pulmaquin in patients with non-CF PA-infected bronchiectasis was evaluated (NCT02104245 and NCT01515007). The results showed that when compared to the placebo group, the Pulmaquin group had an obvious antibacterial effect, higher safety and better tolerance [172].

PA is resistant to various antibiotics. Studies have shown that quorum sensing inhibitors (QSIs) can help improve the anti-infection effect of antibiotics by inhibiting the biofilm formation of PA. Certain researchers prepared self-assembled NPs of newly synthesized squalenyl hydrogen sulfate NPs. This nanocarrier has a significant high loading capacity for tobramycin and the new lipophilic QSI. The prepared tobramycin NPs enhanced the permeability of the biofilm and effectively inhibited the formation of a PA biofilm [173]. The retention and penetration of nanodrugs in bacterial biofilms are still facing significant challenges. Certain researchers developed

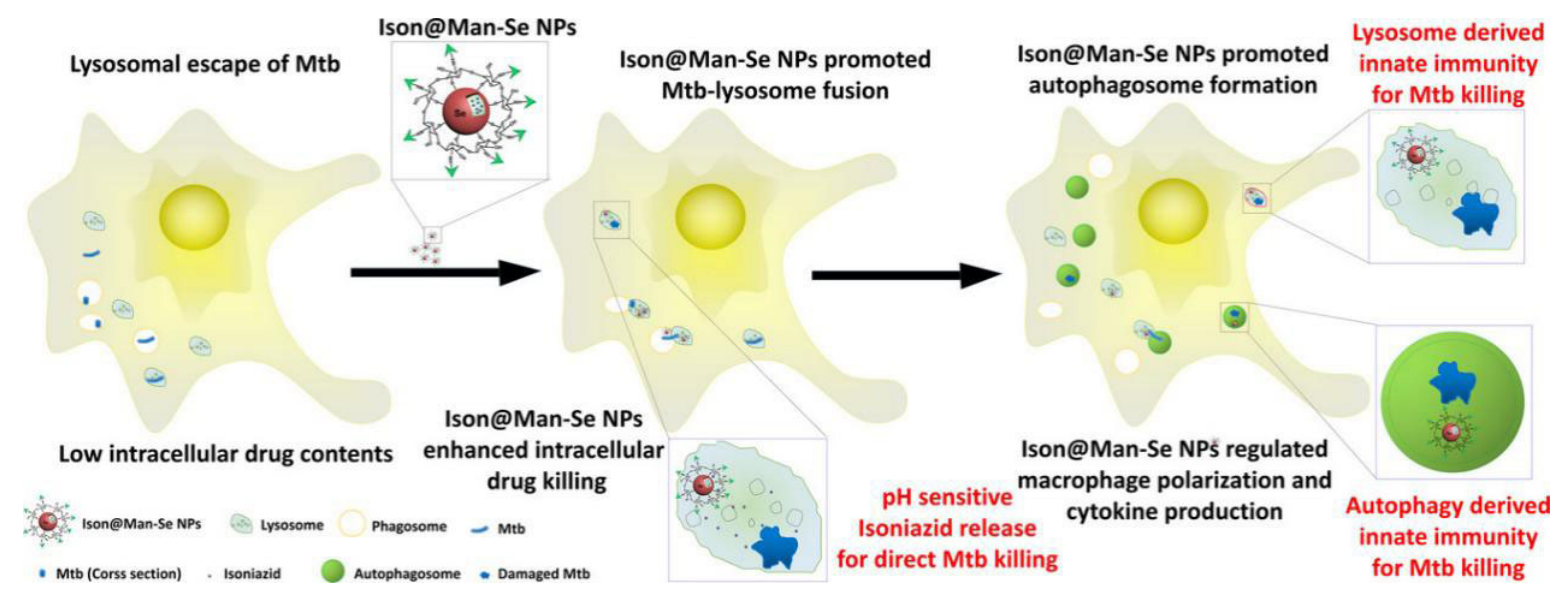

Figure 6 Ison@Man-Se NPs-assisted anti-TB strategy for synergistic killing of intracellular Mtb. Reproduced with permission from Ref. [158], (c) Wiley-VCH Verlag GmbH \& Co. KGaA, Weinheim, 2019. 
azithromycin (AZM)-conjugated cluster NPs (denoted as AZMDA NPs), which can disassemble and release secondary AZMconjugated poly(amidoamine) (PAMAM) NPs (PAMAM-AZM NPs) in the acidic biofilm microenvironment. PAMAM-AZM NPs increased the permeability and retention of the drug in the biofilm, showing significant antibiofilm activity [174].

A ROS-responsive material, 4-(hydroxymethyl) phenylboronic acid pinacol ester-modified $a$-cyclodextrin (Oxi-aCD), was employed to encapsulate moxifloxacin (MXF), generating ROS-responsive MXF-containing NPs (MXF/Oxi-aCD NPs). 1,2-Distearoyl-sn-glycero-3-phosphoethanolamine (DSPE)-PEGfolic acid and DSPE-PEG were coated on the surface of MXF /Oxi-aCD NPs. In vitro studies showed that the anti-PA effect of MXF/Oxi-aCD NPs was higher than that of free MXF. In addition, when compared to the nontargeted MXF/Oxi- $\alpha \mathrm{CD}$ NPs, folate-modified MXF/Oxi- $\alpha \mathrm{CD}$ NPs can effectively target bacterial-infected macrophages and significantly eliminate bacteria in macrophages. When compared to the free MXF and nontargeted MXF/Oxi- $\alpha \mathrm{CD}$ NPs, folate-modified targeted $\mathrm{MXF} / \mathrm{Oxi}-\mathrm{\alpha CD}$ NPs demonstrated a stronger anti-infective effect in mice infected with PA. It should be noted that the survival time of mice was prolonged by the use of targeted $\mathrm{MXF} / \mathrm{Oxi}-\alpha \mathrm{CD}$ NPs. This research strategy provides a direction for overcoming the mucus barrier, achieving controlled release of the drug, and improving drug targeting [175]. Researchers developed a type of sharp acid-sensitive segment of poly $(\beta$-amino ester) NPs with stimulation response, which are coated with PEG biotin on the surface and coated with anti-intercellular adhesion molecule 1 antibody to achieve lung targeting and expand circulation. In a mouse model to analyze acute lung injury, 2-[(aminocarbonyl)amino]-5-(4-fluorophenyl)3-thiophenecarboxamide-loaded NPs targeted the inflammatory site of the lung, induced drug release in the acidic environment, and reduced lung inflammation and injury [176].

\subsubsection{Fungus infections}

In addition to bacteria, fungi are common pathogens in lung infections. Aspergillus is a type of fungal pathogen that can often cause lung infections. Amphotericin B-polymethacrylic acid NPs are utilized against Aspergillus. Through in vivo experiments, it was determined that NPs are not toxic to macrophages derived from lung epithelial cells or monocytes. Aerosol administration can effectively deliver NPs to the lungs and can prevent fungal growth and lung inflammation. The results showed that the killing rate of NPs against Aspergillus was $>99 \%$, and lung tumor necrosis factor- $\alpha$ was reduced by $90 \%$. This study delivered NPs to the lungs safely and effectively through an aerosol route and prevented fungal infections, thus providing a stepping stone for future clinical trials [177]. Certain scholars prepared a type of itraconazole (ITZ) chitosan-based nanopreparation. The prepared nanodrug can be administered orally and has targeting property. The results showed that the nanodrug could significantly kill Cryptococcus neoformans in lung infection foci, thus improving the survival rate of infected mice [178]. Studies have shown that biodegradable ITZ-loaded d-a-tocopheryl PEG 1000 succinate-b-poly(e-caprolactoneran-glycolide) NPs (designed as ITZ-loaded tripolyphosphate (TPP) NPs) can improve the antifungal efficacy. The drugloading rate of ITZ-loaded TPP NPs was high (95\%); moreover, the drug release time was prolonged. The high concentration $(25 \mathrm{mg} / \mathrm{ml})$ of nanodrugs had no cytotoxicity to fibroblasts and HeLa cells. Through in vitro and in vivo experiments, it was determined that ITZ-loaded TPP NPs show high antifungal activity [179].

\subsubsection{Virus infections}

\subsubsection{Influenza virus}

Certain researchers synthesized pulmonary surfactant (PS)biomimetic liposomes that encapsulates 2,3'-cyclic guanosine monophosphate-adenosine monophosphate (cGAMP). The PS-GAMP can mimic the early stages of viral infection and enhance the immune response of mouse humoral and cluster of differentiation 8 (CD8) T cells induced by the influenza vaccine without excessive inflammation. The nasal immunization of PS-GAMP-adjuvanted H1N1 vaccine for two days can produce strong cross-protection for long-distance H1N1 and heterotypes $\mathrm{H} 3 \mathrm{~N} 2, \mathrm{H} 5 \mathrm{~N} 1$, and $\mathrm{H} 7 \mathrm{~N} 9$ viruses for at least six months, while maintaining memory CD8 T cells in the lung. Moreover, the effectiveness of the preparation was verified on a ferret. The detailed process is illustrated in Fig. 7 [180].

Most of the virus vaccines against influenza cannot produce

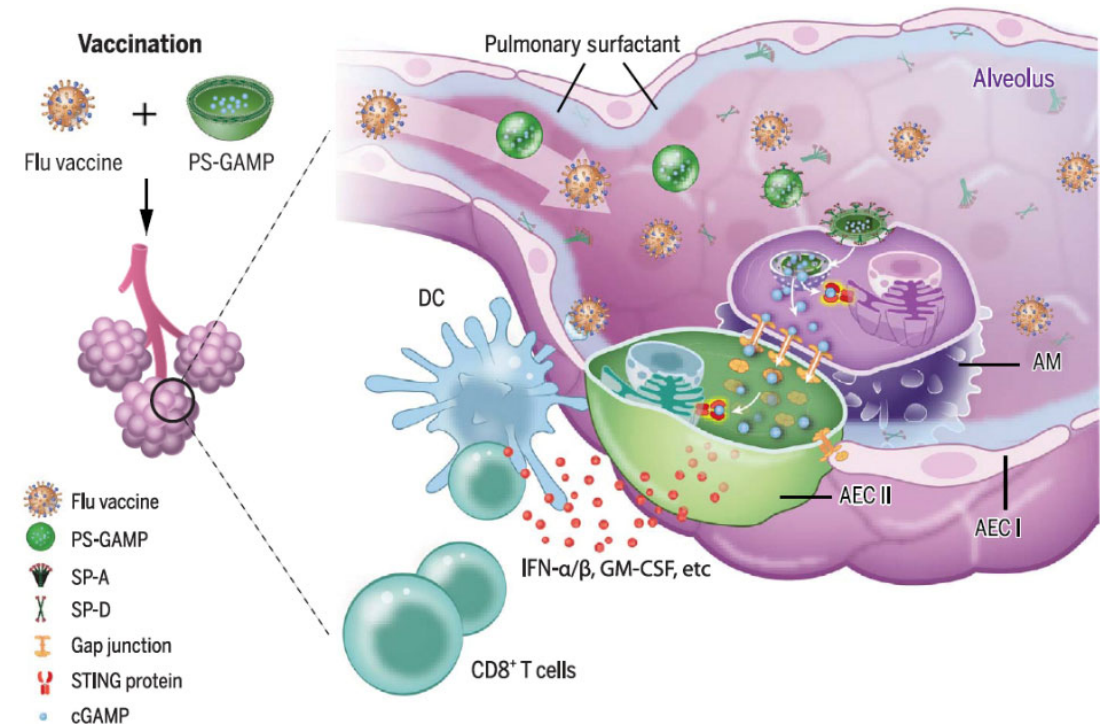

Figure 7 PS-GAMP mediated adjuvanticity. In alveoli, PS-GAMP associates with SP-A or SP-D before entering AMs by means of SP-A or SP-D mediated endocytosis. cGAMP is subsequently released into the cytosol and fluxes into AECs by way of gap junctions. It then activates STING in these cells, resulting in the vigorous production of type 1 immune mediators. These mediators facilitate the recruitment and differentiation of CD11b+ DCs, which in turn direct robust antiviral CD8+ T cell and humoral immune responses. Reproduced with permission from Ref. [180], @ American Association for the Advancement of Science, 2020 
strong immunity in the lung mucosa, which requires further improvement. Studies have shown that adding Ag NPs to the inactivated influenza vaccine can reduce the viral load of influenza mouse models and prevent excessive lung inflammation after influenza virus infection. In addition, Ag NPs increase the titer of plasma cells and specific immunoglobulin A (IgA) antibody, which is a marker of mucosal immunity. In addition, vaccination in the presence of Ag NPs protected mice from lethal influenza. Ag NPs can produce stronger antigen-specific $\operatorname{IgA}$ with lower toxicity by promoting the regeneration of bronchus-associated lymphoid tissue. This strategy provides directions for improving the efficacy of influenza vaccines in the future [181].

\subsubsection{Middle East respiratory syndrome coronavirus}

Middle East respiratory syndrome coronavirus (MERS-CoV) is a highly infectious respiratory virus. The outbreak of a pandemic requires rapid and accurate diagnostic methods, which is significantly important for the prevention and control of infectious diseases. A colorimetric method based on the extended form of the double-stranded DNA self-assembly shielded by GNPs was developed for the detection of MERS-CoV. The shift in localized surface plasmon resonance and color change of GNPs were used to verify the existence of virus molecules in the ultraviolet-visible wavelength range. The colorimetric method detected $30 \mathrm{bp}$ of MERS-CoV for a concentration as low as $1 \mathrm{pmol} / \mu \mathrm{L}$ and has high detection efficiency. This strategy provides direction for the development of other infectious disease detection methods [182]. Certain researchers developed an immunosensor for detecting MERS-CoV. The detection mechanism of this immunosensor is a competitive analysis based on a GNP-modified carbon array electrode. Array electrodes can multiplex detection of different types of coronaviruses (such as influenza A, influenza B). It has the advantages of simplicity, sensitivity, and accuracy. This technique has been used for the detection of nasal samples [183]. Researchers have also developed a new NP vaccine against MERS-CoV for the delivery of a subunit of stimulator of interferon (IFN) gene agonists and viral antigens. In a mice model immunized with MERS-CoV NP vaccine candidates, the vaccine showed therapeutic effectiveness by stimulating effective antigen-specific $\mathrm{T}$-cell responses and neutralizing antibodies. In the MERS-CoV permissible transgenic mouse model, the mice immunized with the vaccine could be protected from fatal attacks by the MERS-CoV and could avoid eosinophilic immune pathological reactions. The biocompatible hollow NPs described by the institute provide an excellent strategy for the delivery of subunit candidate vaccines and novel adjuvants, thus providing broad prospects for accelerating the development of vaccines against certain infectious diseases [184].

\subsubsection{Novel coronavirus}

A novel coronavirus, called severe acute respiratory syndrome coronavirus 2 (SARS-CoV-2), which can cause severe pneumonia, was discovered in Wuhan, China in December 2019. It was named coronavirus disease 19 (COVID-19) by WHO. COVID-19 is highly infectious and lethal [185]. As a new and highly contagious respiratory infection disease, COVID-19 is spreading worldwide. COVID-19 is caused by SARS-CoV-2 infection and is primarily spread through contact, respiratory droplets, and feces [186]. SARS-CoV-2 belongs to genus $\beta$ and subgenus sarbe of the coronaviridae family [187]. SARS-CoV-2 has a similar structure as MERS-CoV and severe acute respiratory syndrome coronavirus (SARS-CoV). It is composed of spike glycoprotein (S protein), hemagglutination acetyl esterase granule protein, membrane glycoprotein, small molecule envelope glycoprotein, glycocapsid phosphate protein, and RNA. Studies have confirmed that angiotensin-converting enzyme 2 (ACE2) is the host cell receptor of the SARS-CoV-2 [187]. The S protein performs an important role in the process of recognizing and binding host cell surface receptors and mediating the fusion of virus envelope and cell membrane. The $S$ protein can infect human respiratory epithelial cells by binding to human ACE2 protein $[188,189]$.

Currently, there is no specific drug for COVID-19. Clinical trials are being conducted in several countries to develop antiviral drugs or vaccines. Several drugs approved for other diseases are also being evaluated for the treatment of COVID-19. Almost all of these drugs have the effect of preventing the virus from entering the host cell, preventing the virus from replicating and infecting other cells, or potentially inhibiting the activity of proteases (such as lopinavir/ritonavir antiviral drugs). Given the possibility of the continuous evolution of virus strains, the development of treatments for COVID-19 has become more challenging. Nanotechnology-based methods to help combat COVID-19 may have good prospects. Nanotechnology can provide a variety of strategies, including (1) using nano-delivery system to deliver therapeutic components to target lesion sites, (2) development of nanotechnology detection methods for rapid and sensitive detection, (3) preparation of ultra-fine filters for masks or hemodialysis, (4) preparation of surface coatings that can inactivate viruses to resist virus adhesion.

\subsubsection{NP-based detection methods}

Currently, the basis for determining the COVID-19 infection is primarily based on the detection results obtained using reverse transcriptase polymerase chain reaction (RT-PCR) for oropharyngeal and/or nasopharyngeal swabs [190]. A colorimetric assay based on GNPs, when capped with suitably designed thiol-modified antisense oligonucleotides (ASOs) specific to the nucleocapsid phosphoprotein of SARS-CoV-2, can be used for diagnosing positive COVID-19 cases within 10 min from the isolated RNA samples. Thiol-modified ASOcapped GNPs can selectively aggregate in the presence of SARS-CoV-2 target RNA sequence. After adding ribonuclease $\mathrm{H}$, a visible and detectable precipitate is formed. This method does not require any complicated instrument or technology and can detect COVID-19 selectively and intuitively [191].

A sensitive and rapid lateral flow immunoassay (LFIA) was developed for the detection of anti-SARS-CoV-2 immunoglobulin $\mathrm{G}$ (IgG) in human serum based on lanthanide-doped polystyrene NPs. Twelve RT-PCR negative samples and seven RT-PCR positive samples were selected. The results of LFIA showed that one of the RT-PCR negative samples was positive, and the other samples were consistent with the results of RT-PCR. It can be observed that the detection method can achieve sensitive and rapid detection of anti-SARS-CoV-2 IgG, and can be used for positive identification of suspicious cases [192]. One study showed certain preliminary ideas for monitoring the existence of SARS-CoV-2 using nanotechnology. The detection mechanism was similar to that of enzyme-linked immunosorbent assay. The antibody was coupled with GNPs and then formed a tertiary complex with virus antigen. Then, the detection results were analyzed based on the color change of GNPs on the antibody [193]. Nano biosensor is a type of laboratory equipment used for clinical and environmental analysis [194]. It utilizes the excellent optical and electrical properties of nanomaterials and combines them with related receptors to selectively detect 
samples. Studies have used the electrocatalytic properties of GNPs for hydrogen evolution to detect sample cells (such as cancer cells) [195]. This nanobiosensor is based on the reaction of specific antibodies coupled to GNPs and cell surface proteins. Using the antigen-antibody reactions, similar strategies can also be applied to virus detection [196]. Recently, a field-effect transistor sensor (modified with a graphene sheet) was prepared to detect SARS-CoV-2. The detection mechanism is primarily based on the binding reaction of SARS-CoV-2 S protein and its specific antibodies [196].

\subsubsection{NP-based therapy}

It is significantly important to prevent the virus from entering the host cell and to inhibit the replication of the virus for the prevention and control of viral infectious diseases. Based on the advantages of nanotechnology, this technology may have broad prospects in the treatment of COVID-19. According to reports, certain nanomaterials (such as GNPs and carbon nanomaterials) can interact with viruses and prevent viruses from entering cells [197]. A study showed that boric acid conjugated carbon QD-based NPs can inhibit human immunodeficiency virus (HIV) from entering cells by inhibiting the formation of syncytia [198]. Another study also showed that carbon QDs coupled with boric acid ligands can interfere with the function of coronavirus S protein, thereby preventing it from entering the host cell [199]. Figure 8 illustrates the nanomedicine strategies for COVID-19 prevention and treatment.

\subsubsection{Spike glycoprotein}

Understanding the pathogenesis of COVID-19 is the key to the development of COVID-19 therapy. It is reported that SARS-CoV-2 and MERS-CoV share the same membrane protein, $S$ protein [200]. The $S$ protein controls the binding of the MERS-CoV to the host cell through the dipeptidyl peptidase-4 receptor [201]. Therefore, the virus can enter the host cell through the $S$ protein and cause the disease. Inhibition of virus fusion with host cells is an effective strategy for the treatment of COVID-19.

Studies have shown that Matrix-M1 adjuvant combined with recombinant MERS-CoV S NP vaccine can effectively inhibit MERS-CoV replication. In the study, it was observed that high-titer anti-S neutralizing antibodies were produced in mice, indicating that the MERS-CoV S NP vaccine has the function of resisting MERS-CoV infection [202]. This study proves once again that the coupling of a nanovaccine and $S$ protein has a potential therapeutic effect, which is worthy of an in-depth study. This study provides a strategy for SARS-CoV-2 vaccine development because both SARS-CoV-2 and MERS-CoV have the $S$ protein.

\subsubsection{Angiotensin-converting enzyme 2}

According to the sequencing results of the SARS-CoV-2 genome, the key genes are considerably similar to other coronaviruses (such as SARS-CoV) [187, 203]. The primary binding target of SARS-CoV is the human cell surface receptor ACE2, which performs a key role in SARS-CoV fusion with host cells [187, 204-206]. This discovery provides a direction for the development of drugs against SARS-CoV-2. For example, we can prepare effective biological inhibitors to block the interaction between SARS-CoV-2 and ACE2 receptors, thus preventing the virus from entering cells. Considering the importance of S1 protein in cell uptake, several researchers are exploring the possibility of S1 as the primary target of the vaccine. Nanotechnology may provide an effective strategy for antiviral therapy. Certain nanotechnology-based therapeutic platforms have achieved gratifying results in preclinical studies, and have successfully demonstrated antiviral effects on some human viral pathogens (such as HIV, human papillomavirus, herpes simplex virus, and respiratory syncytial virus) [193, 207-209]. The utilization of nanotechnology to fight viruses adds hope to the development of safe and effective broadspectrum antiviral drugs.

\subsubsection{Adaptor-associated protein kinase 1}

Studies have shown that all target cells of SARS-CoV-2 have one thing in common, i.e., in all cells, the adaptor-associated protein kinase 1 (AAK1) is associated with adaptor protein complex 2, which is a key regulator of endocytosis. Although there is no research on the inhibition of AAK1 to prevent SARS-CoV-2 from entering target cells, there have been reports on the use of AAK1 inhibitors to prevent the endocytosis of other viruses. A researcher conducted a simulation study and believed that AAK1 inhibitors may inhibit the virus from entering target cells [210]. Inhibitors can exert better effects at high doses, but they can also cause serious toxicity and side effects [211]. Fortunately, not all AAK1 inhibitors cause serious adverse reactions. Another study showed that pacritinib, a Janus kinase inhibitor, combined with another modulator of endocytosis (cyclin G-related kinase) can inhibit AAK1, thereby preventing endocytosis virus [212]. Researchers developed a method of erlotinib-conjugated liposomes based on the DDS strategy for the treatment of lung cancer. The in vivo study showed that the nanoformulation could significantly improve drug biodistribution, enhance drug targeting after administration,

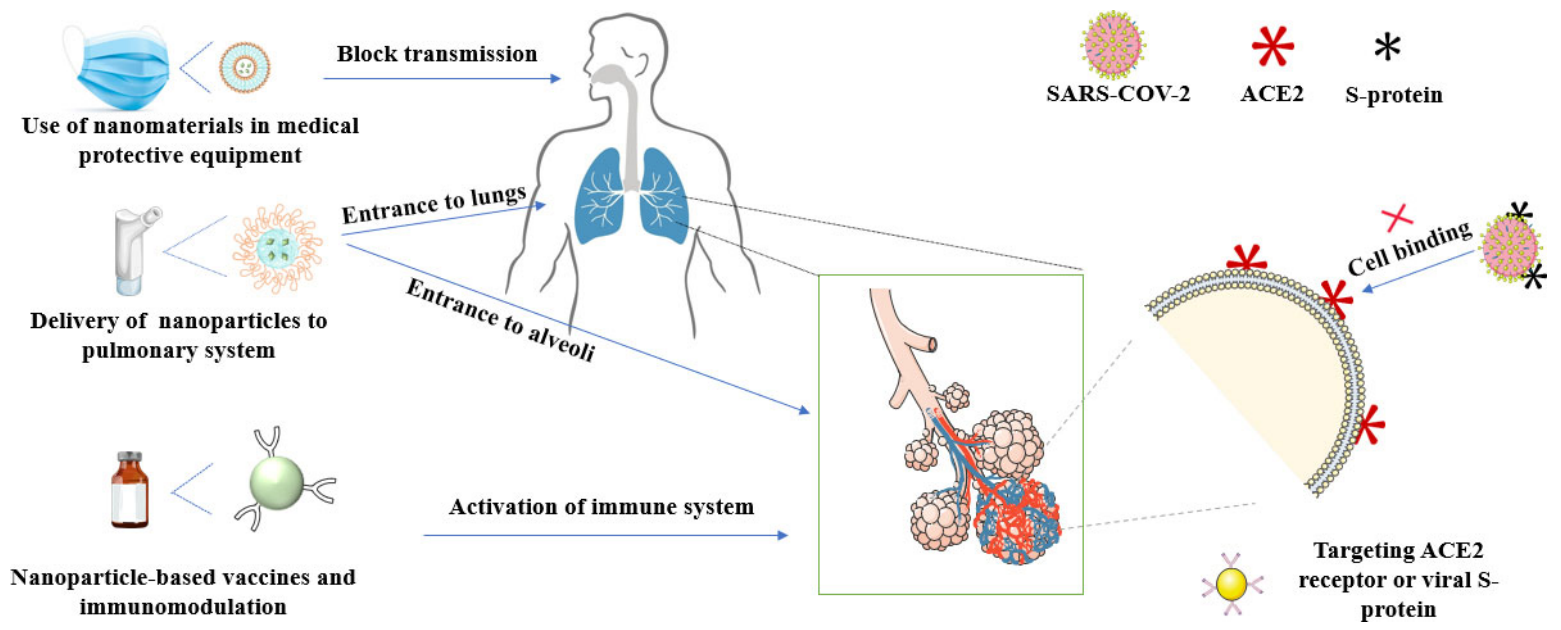

Figure 8 Nanomaterials for prevention and therapy of COVID-19. 
and significantly increase the relative bioavailability of the drug, and can be released quickly and continuously within the first $4 \mathrm{~h}$ after administration of the drug [213]. If it can be verified that the drugs related to AAK1 can inhibit SARSCoV-2 from entering the target cells, and that nanotechnology is used to optimize the related drugs, it is expected that these will be used for the treatment of COVID-19.

\subsubsection{Messenger RNA}

The mRNA is a promising vaccination platform. By analyzing the gene expression of the untranslated region (UTR) of mRNAs, researchers determined that the best combination was of 3'UTR and 5'UTR, which was named NASAR. Studies have shown that NASAR mRNA-loaded lipid NPs can express the corresponding products in vitro and in vivo. This study provides a direction for the development of SARS-CoV-2 vaccine [214].

\subsection{Asthma}

Asthma is a common lung disease characterized by bronchial hyperresponsiveness, chronic airway inflammation, and intermittent reversible airway obstruction [184]. Common treatment drugs include bronchodilators and inhaled corticosteroids. The current therapeutic drugs have certain limitations, especially corticosteroids, which can cause multiple serious side effects and increase mortality. In recent years, nanotechnology has been considerably developed. Several studies have confirmed that drug delivery through the nano-delivery systems can improve the efficacy of drugs [46].

\subsubsection{NP-based detection method}

An electronic nose based on nanosensors was developed. It can detect specific volatile organic compounds in exhaled gas in a noninvasive manner [215]. Previous clinical studies demonstrated that the method can distinguish between the exhaled breaths of asthma patients and healthy people, and can judge the severity of asthma [216]. Electronic nose technology can also be used to determine the type of asthma. Asthma has four inflammatory phenotypes, and the diagnosis of asthma phenotype is significantly important for the personalized treatment of asthma [217]. Electronic noses have been used to perform high-precision, noninvasive assessments to distinguish patients with eosinophils, neutrophils, and polynuclear granulocyte asthma phenotypes. A clinical trial (NCT02026336) evaluated the efficacy of the method, and the results showed that the different phenotypes of inflammatory asthma could be easily distinguished by the respiratory chart of the electronic-nasal device [218].

\subsubsection{Nanodrugs}

\subsubsection{Hormone NPS}

Inhaled medication is essential in the treatment of asthma; moreover, dry powder inhalers are the most common type at present. Recently, certain scholars developed nano-embedded microparticle (NEM) powders. The results show that the spray-dried NEM formulations containing nucleic acids have the best properties currently known for inhalation therapy [219]. Local administration is significantly important in the treatment of asthma, and nanotechnology can be used to improve drug delivery efficiency. A study showed that the inhalation of dry powder NPs can improve delivery efficiency and enhance lung permeability of the drug [220]. In addition, there are also studies showing that, in mouse models with asthma, when compared to free steroids, steroids encapsulated in NPs have better efficacy at the site of airway inflammation
[221]. NPs can efficiently deliver therapeutic drugs to diseased tissues, improve the deposition of drugs in the lungs, and thus enhance the therapeutic effect. Beclomethasone dipropionate was delivered to the lung by PEGylated PAMAM dendrimer, which can increase the drug accumulation in the lung, improve the bioavailability of the drug, and reduce the dosage and side effects of the drug. The study also confirmed that a nanocarrier can effectively transport dexamethasone to the lung, thus avoiding allergic lung inflammation, reducing the production of eosinophils, and reducing the secretion of inflammatory cytokines [222]. When compared to free dexamethasone, dexamethasone NPs can reduce airway hyperresponsiveness in a superior manner [223].

\subsubsection{Salbutamol NPS}

Salbutamol is a commonly used bronchodilator in asthma patients. By modifying salbutamol into NPs, studies have shown that, when compared to conventional dry powder inhalation formulations, the total deposition of salbutamol NPs in the lungs increased by 2.3 times. Long-term remission of bronchospasm can be achieved by maintaining the effective drug concentration at the target site for a long time. When compared to micronized salbutamol, the effect of NP delivery of salbutamol in the pharyngeal population was reduced, showing better peripheral deposition, thus indicating that NPs have better penetration and bioavailability and have a longer drug-maintenance time [220]. Another study demonstrated that salbutamol liposome NPs can prolong the retention time in the lesion, and its curative effect is better than that of the free drug [224]. Certain researchers encapsulated salbutamol into vesicles (liposomes). Preclinical studies have shown that salbutamol can achieve controlled release. A phase I clinical trial was conducted to study the sustained release and relative bioavailability of salbutamol NPs after inhalation (NCT03059017) [225].

\subsubsection{Others}

Studies have shown that $\mathrm{T}$ helper type 2 (Th2) cytokines can be used as the primary target of new anti-asthma drugs. Researchers also determined that bavachinin can selectively inhibit the production of Th2 cytokines, which demonstrated a significantly good effect in a mouse model with asthma. Given the low water solubility of atorvastatin, the researchers developed a NP delivery system for oral atorvastatin. Bavachininloaded PEG5000-PLGA NPs were orally administered, and a significantly superior anti-asthma effect was observed in an asthmatic mouse model. Histological analysis of lung slices revealed that Th2 cytokine production was reduced in the nanodrug treatment group [226]. Andrographolide (AG) has anti-asthmatic effects and has lower toxicity and fewer side effects than glucocorticoids; however, its bioavailability is lower and plasma half-life is shorter. To overcome these defects, some researchers encapsulated AG in NPs (AGNP) for anti-asthma treatment. After oral/pulmonary administration to a murine asthma model, AG nanoformulation bioavailability was significantly improved, and the secretion of inflammatory factors was also relatively decreased. The results of the study also show that the pulmonary route of administration has a better therapeutic effect than oral administration [227]. Nanoimmunotherapy, where specific antigens that induce asthma are targeted, has broad research prospects. Researchers have developed a nanovaccine for dust-mite allergic asthma and demonstrated a good therapeutic effect. The results also show that the particle size of the NP vaccine used for vaccination performs an important role in preventing allergies caused by house dust mites [228, 229]. Chemokines signal 
transduction through the CC chemokine receptor 3 (CCR3) is a major factor in the development of treatments for asthma. Researchers synthesized a novel peptide NP CCR3 inhibitor (R321) that can block CCR3 signal transduction. The study showed that R321 could effectively prevent eosinophils from collecting into the airway, lung, and blood, and prevent the occurrence of airway hyperresponsiveness in asthmatic mice. The detailed process is illustrated in Fig. 9 [230].

In recent years, gene therapy has performed an important role in the treatment of several diseases and has become one of the trending topics in the medical field. Gene therapy with nanotechnology may be an effective treatment for asthma. Studies have shown that chitosan-IFN- $\gamma$-pDNA NPs (CIN) can significantly reduce airway hyperresponsiveness induced by ovalbumin (OVA)-induced allergic asthma in mice [231]. In addition, researchers also identified that CIN can inhibit the release of pro-inflammatory factors from OVA-specific CD8+ T lymphocyte population and inhibit the activation of dendritic cells. They speculated that the mechanism of CIN in the treatment of allergic asthma may be related to $\mathrm{T}$ helper type 1/Th2 immunoregulation [232].

\section{Challenges and strategies in NP delivery} systems for diagnosis and treatment of pulmonary

\section{diseases}

Table 1 summarizes the application progress of nanomedicine in lung diseases in recent years, from which we can see that nanomedicine has an important clinical research value in the diagnosis and treatment of lung diseases. As a frontier science, nanomedicine has played an important role in the management of many other diseases and also provides new strategies to overcome the current difficulties in the diagnosis and treatment of lung diseases.

The field of NP drug delivery has developed significantly, providing several strategic options for the preparation of DDS [233, 234]. Nano-delivery systems have been widely used in drug delivery or imaging. However, owing to various reasons (such as complicated manufacturing), they often face the dilemma of research failure; moreover, several nanodrugs cannot be used in clinical practice. This is because reproducibility is poor, mass production is difficult, and this advanced therapy may be more expensive than existing therapies $[52,235]$.

Lung delivery strategies also face several challenges. The following factors may affect the effectiveness of NPs in lung delivery: (1) particle size, (2) clearance of NPs by mucosal cilia, (3) durability of shear resistance of NPs during atomization, (4) uptake of NPs by alveolar macrophages, (5) targeting ability of NPs, (6) steric hindrance and adhesion between NPs and drug delivery channels, and (7) degradation of nanodrugs by proteases produced in vivo [236, 237]. For example, certain researchers administered nanomedicines by inhalation to treat fluoride-related bacterial infections; however, the results of the study show that the treatment effect is low, primarily as the viscosity of sputum is much larger than that of water $[237,238]$. In addition, NPs may also be unstable. Factors such as disintegration and/or biodegradation of NPs, leakage of loaded drugs, and dissociation of surface-modified components can affect the stability of NP delivery systems [239]. When compared to free drugs, nanodrugs have the advantages of targeted and controlled release, but when they are deposited in the lungs,

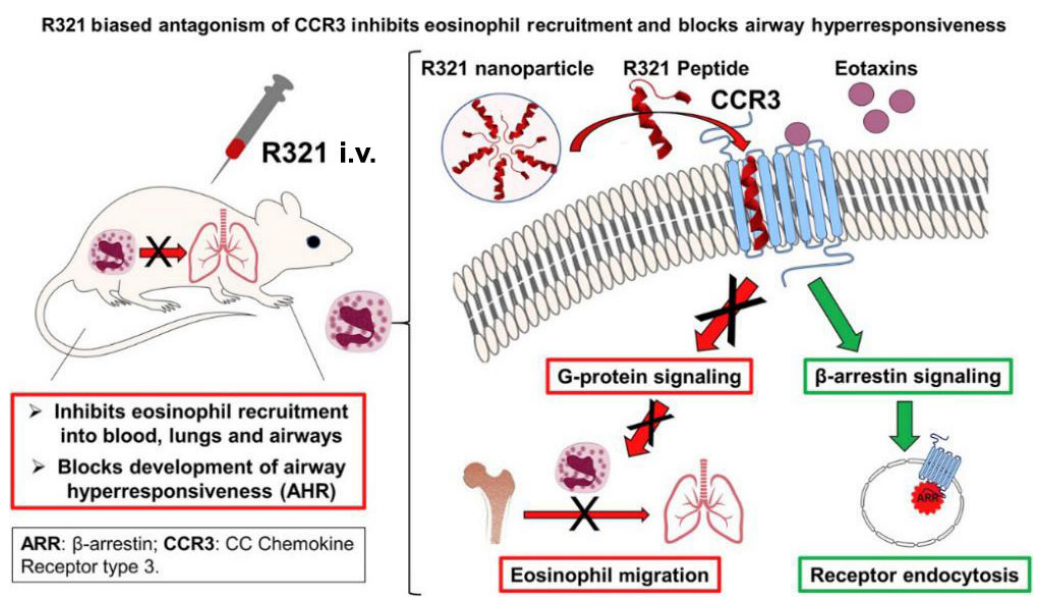

Figure 9 Novel peptide nanoparticle-biased antagonist of CCR3 blocks eosinophil recruitment and airway hyperresponsiveness. Reproduced with permission from Ref. [230], ๔ American Academy of Allergy, Asthma \& Immunology, 2018.

Table 1 Clinically available and in (pre-)clinical development nanomedicines for lung diseases

\begin{tabular}{|c|c|c|c|c|}
\hline Particle type & Active pharmaceutical ingredient(s) & Indication & Development stage & Ref. \\
\hline PEGylated liposome & Doxorubicin & Lung cancer & Market & [66-69] \\
\hline Polymeric & Cisplatin (CDDP) and DOX & Lung cancer & Pre-clinical & {$[70]$} \\
\hline Polymeric & Irinotecan & Lung cancer & Pre-clinical & [71] \\
\hline Polymeric & Irinotecan & Lung cancer & phase I & {$[72]$} \\
\hline Polymeric & Irinotecan & Lung cancer & phase II & [73] \\
\hline Liposome & Irinotecan & Lung cancer & phase I/II & {$[74,75]$} \\
\hline Micellar & PTX & Lung cancer & Market & [77] \\
\hline Polymeric & PTX & Lung cancer & Market & [78] \\
\hline Polymeric & PTX & Lung cancer & Pre-clinical & {$[80]$} \\
\hline Polymeric & CDDP & Lung cancer & Pre-clinical & {$[84]$} \\
\hline Polymeric & CDDP & Lung cancer & Pre-clinical & [85] \\
\hline
\end{tabular}




\begin{tabular}{|c|c|c|c|c|}
\hline Particle type & Active pharmaceutical ingredient(s) & Indication & Development stage & Ref. \\
\hline Liposome & CDDP & Lung cancer & Pre-clinical & {$[86]$} \\
\hline Liposome & AFT & Lung cancer & Pre-clinical & {$[88]$} \\
\hline GNPs/ Polymeric & siRNA & Lung cancer & Pre-clinical & [91] \\
\hline Polymeric & $5 \mathrm{FU}$ & Lung cancer & Pre-clinical & {$[92]$} \\
\hline Polymeric/silica & siRNA & Lung cancer & Pre-clinical & [93] \\
\hline Polymeric & PTX & Lung cancer & phase I & [96-98] \\
\hline Non-viable minicells & miRNA & Lung cancer & phase I & {$[100,101]$} \\
\hline Liposome & miR-660 (CCL660) & Lung cancer & Pre-clinical & {$[102]$} \\
\hline Liposome & MUC1 & Lung cancer & phase III & {$[104]$} \\
\hline Liposome & MUC1 & Lung cancer & phase III & {$[105,106]$} \\
\hline Polymeric & Gene encoding CFTR & CF & Pre-clinical & [109] \\
\hline Liposome & Aerosolized PGM169 plasmid DNA encoding the CFTR gene & $\mathrm{CF}$ & phase IIb & {$[114]$} \\
\hline Liposome & Chemically modified CFTR mRNA (cmCFTR) & $\mathrm{CF}$ & Pre-clinical & {$[115]$} \\
\hline Polymeric & Human CFTR (hCFTR) & $\mathrm{CF}$ & Pre-clinical & [116] \\
\hline Liposome & siRNA & $\mathrm{CF}$ & Pre-clinical & [117] \\
\hline Polymeric & Glycosaminoglycan-binding enhanced transduction (GET) & $\mathrm{CF}$ & Pre-clinical & [118] \\
\hline Liposome & Lumacaftor and ivacaftor & $\mathrm{CF}$ & Pre-clinical & {$[120]$} \\
\hline Liposome & $\mathrm{AM}$ & $\mathrm{CF}$ & Market & {$[164]$} \\
\hline Liposome & Ciprofloxacin & $\mathrm{CF}$ & Phase II & {$[121,122]$} \\
\hline Polymeric & Ciprofloxacin & $\mathrm{CF}$ & Pre-clinical & [123] \\
\hline Polymeric & Tobramycin & $\mathrm{CF}$ & Pre-clinical & {$[124]$} \\
\hline Liposome & $\mathrm{AM}$ & COPD & Pre-clinical & {$[134]$} \\
\hline Polymeric & $\mathrm{AM}$ & COPD & Pre-clinical & {$[136]$} \\
\hline Liposome/Polymeric & $\mathrm{MnPD}$ and $\mathrm{pHDAC} 2$ & COPD & Pre-clinical & [137] \\
\hline Liposome & miR-146a & COPD & Pre-clinical & {$[138]$} \\
\hline Polymeric & Ibuprofen & COPD & Pre-clinical & {$[142]$} \\
\hline $\mathrm{ZnO}$ and $\mathrm{Ag}$ & Rifampicin & TB & Pre-clinical & {$[155]$} \\
\hline Polymeric & Isoniazid & $\mathrm{TB}$ & Pre-clinical & {$[156]$} \\
\hline Particle type & Active pharmaceutical ingredient(s) & Indication & Development stage & Ref. \\
\hline Polymeric & Combinations of anti-TB drugs & TB & Pre-clinical & [157] \\
\hline $\mathrm{Se}$ & Isoniazid & $\mathrm{TB}$ & Pre-clinical & {$[158]$} \\
\hline Liposome & $\mathrm{AM}$ & Pneumonia & Market & [164-167] \\
\hline Liposome & Ciprofloxacin & Pneumonia & Pre-clinical & [169-171] \\
\hline $\begin{array}{l}\text { Synthesized squalenyl } \\
\text { hydrogen sulfate NPs }\end{array}$ & Tobramycin & Pneumonia & Pre-clinical & {$[173]$} \\
\hline Polymeric & Azithromycin & Pneumonia & Pre-clinical & {$[174]$} \\
\hline Polymeric & Moxifloxacin (MXF) & Pneumonia & Pre-clinical & {$[175]$} \\
\hline Polymeric & $\begin{array}{l}\text { 2-[(aminocarbonyl)amino]-5-(4-fluorophenyl)-3-thiophenec } \\
\text { arboxamide }\end{array}$ & Pneumonia & Pre-clinical & {$[176]$} \\
\hline Polymeric & Amphotericin B & Pneumonia & Pre-clinical & {$[177]$} \\
\hline Polymeric & Itraconazole & Pneumonia & Pre-clinical & {$[178]$} \\
\hline Polymeric & Itraconazole (ITZ) & Pneumonia & Pre-clinical & [179] \\
\hline Liposome & cGAMP & Pneumonia & Pre-clinical & {$[180]$} \\
\hline $\mathrm{Ag}$ & Inactivated influenza vaccine & Pneumonia & Pre-clinical & {$[181]$} \\
\hline Polymeric & Nucleic acid & Asthma & Pre-clinical & [219] \\
\hline Nano-SBS & Salbutamol & Asthma & Pre-clinical & {$[220]$} \\
\hline Polymeric & Betamethasone phosphate & Asthma & Pre-clinical & {$[221]$} \\
\hline Polymeric & Beclomethasone dipropionate & Asthma & Pre-clinical & {$[222]$} \\
\hline Polymeric & Dexamethasone & Asthma & Pre-clinical & [223] \\
\hline Liposome & Salbutamol & Asthma & Pre-clinical & [224] \\
\hline Liposome & Salbutamol & Asthma & phase I & [225] \\
\hline Polymeric & Bavachinin & Asthma & Pre-clinical & [226] \\
\hline Polymeric & Cytosine-phosphate-guanine motifs (CpG) & Asthma & Pre-clinical & {$[228,229]$} \\
\hline Polymeric & $\mathrm{R} 321$ & Asthma & Pre-clinical & {$[230]$} \\
\hline Polymeric & IFN- $\gamma$-pDNA & Asthma & Pre-clinical & {$[231,232]$} \\
\hline
\end{tabular}


they may still be rapidly and non-selectively cleared by the mononuclear phagocyte system [240].

When designing a nano-DDS system, it is necessary to further optimize its surface properties, particle size, and particle shape, to better penetrate the pulmonary biological barrier [63]. Ideally, NPs that successfully penetrate the lung barrier should have the following characteristics: (1) small particle size, (2) surface coating with zwitterionic materials or PEG, (3) strong targeting ability, (4) sufficient loading of active drugs, (5) low toxicity, and so on. It is known that the spherical PEGylated NP with particle size less than $200 \mathrm{~nm}$ can be transported at high speed through biological barriers such as biofilm and mucus $[241,242]$. Studies have shown that NPs with small particle size and neutral charge surface can prolong the residence time in vivo [63]. NPs modified with zwitterionic materials or PEG may reduce or lose the possibility of drug loading on the surface of NPs, which primarily depends on the degree of surface modification. In addition, the smaller NPs may also lead to a decrease in drug loading. Therefore, the development of ideal NPs must consider a variety of factors, which requires us to explore better strategies for the preparation of NPs.

\section{Conclusion and future directions}

This review primarily introduced the applications of nanotechnology in the diagnosis and treatment of lung diseases, including COVID-19 and lung cancers. Nanotechnology provides more strategies for the diagnosis and treatment of lung diseases. Although nanotechnology has broad application prospects, it also has several disadvantages. For example, the particle size distribution of nanodrugs is uneven and the reproducibility is insufficient. Besides, commercialization and mass production are temporarily difficult $[243,244]$. In addition, certain studies have shown that the accumulation of nanodrugs in healthy organs and tissues can lead to systemic side effects [245]. Poor biological distribution of nanodrugs can result in poor efficacy and systemic toxicity. The next stage in this development area will be to refine the nanoparticle drug delivery system to make it more effective. Active targeting may provide strategies for improving the biodistribution of nanodrugs $[246,247]$. To further improve the effect of lung-targeted drug delivery, we can determine the receptor that only exists in the lung through elaborate studies. The discovery of this unique receptor will help promote drug action on specific parts of the lung, such as bronchioles and alveoli. Besides, to improve treatment efficiency and reduce systemic toxicity, NPs can be optimized to release drugs through a trigger release or controlled release [248]. Moreover, international standards for in vitro and in vivo models must be developed for effective comparison between studies. Opportunities and challenges coexist; for better application of nanotechnology in the medical field, we must conduct more research work.

We firmly believe that we will witness the remarkable progress of nanotechnology, which will greatly improve the way clinicians manage lung diseases. It will significantly improve the clinical efficacy for patients, especially for those who are currently resistant to conventional drug therapy.

\section{Acknowledgements}

This research was supported by the National Science and Technology Major Project of the Ministry of Science and Technology of China (No. 2018ZX10301402), the National Natural Science Foundation of China (No. 51973243), the International Cooperation and Exchange of the National Natural
Science Foundation of China (No. 51820105004), the Guangdong Innovative and Entrepreneurial Research Team Program (No. 2016ZT06S029), the Guangdong Basic and Applied Basic Research Foundation (No. 2019A1515110903), the Sanming Project of Medicine in Shenzhen (No. SZSM201911004) and the Research Start-up Fund of Post-doctoral, of SAHSYSU (No. ZSQYRSFPD0016).

\section{References}

[1] Azarmi, S.; Roa, W. H.; Löbenberg, R. Targeted delivery of nanoparticles for the treatment of lung diseases. Adv. Drug Deliv. Rev. 2008, 60, 863-875.

[2] Newman, S. P. Drug delivery to the lungs: Challenges and opportunities. Ther. Deliv. 2017, 8, 647-661.

[3] Patton, J. S.; Brain, J. D.; Davies, L. A.; Fiegel, J.; Gumbleton, M.; Kim, K. J.; Sakagami, M.; Vanbever, R.; Ehrhardt, C. The particle has landed-characterizing the fate of inhaled pharmaceuticals. $J$. Aerosol. Med. Pulm. Drug Deliv. 2010, 23 Suppl 2, S71-S87.

[4] Yu, C. P. Exact analysis of aerosol deposition during steady breathing. Powder Technol. 1978, 21, 55-62.

[5] Bray, F.; Ferlay, J.; Soerjomataram, I.; Siegel, R. L.; Torre, L. A.; Jemal, A. Global cancer statistics 2018: GLOBOCAN estimates of incidence and mortality worldwide for 36 cancers in 185 countries. CA Cancer J. Clin. 2018, 68, 394-424.

[6] di Sant'Agnese, P. A.; Davis, P. B. Research in cystic fibrosis (third of three parts). N. Engl. J. Med. 1976, 295, 597-602.

[7] Siafakas, N. M.; Vermeire, P.; Pride, N. B.; Paoletti, P.; Gibson, J.; Howard, P.; Yernault, J. C.; Decramer, M.; Higenbottam, T.; Postma, D. S. et al. Optimal assessment and management of chronic obstructive pulmonary disease (COPD). The European respiratory society task force. Eur. Respir. J. 1995, 8, 1398-1420.

[8] Sevinç, F.; Prins, J. M.; Koopmans, R. P.; Langendijk, P. N. J.; Bossuyt, P. M.; Dankert, J.; Speelman, P. Early switch from intravenous to oral antibiotics: Guidelines and implementation in a large teaching hospital. J. Antimicrob. Chemoth. 1999, 43, 601-606.

[9] MacGregor, R. R.; Graziani, A. L. Oral administration of antibiotics: A rational alternative to the parenteral route. Clin. Infect. Dis. 1997, $24,457-467$

[10] Patton, J. S.; Byron, P. R. Inhaling medicines: Delivering drugs to the body through the lungs. Nat. Rev. Drug Discov. 2007, 6, 67-74.

[11] Rosen, H.; Abribat, T. The rise and rise of drug delivery. Nat. Rev. Drug Discov. 2005, 4, 381-385.

[12] Di Marco, M.; Shamsuddin, S.; Razak, K. A.; Aziz, A. A.; Devaux, C.; Borghi, E.; Levy, L.; Sadun, C. Overview of the main methods used to combine proteins with nanosystems: Absorption, bioconjugation, and encapsulation. Int. J. Nanomedicine 2010, 5, 37-49.

[13] Roger, E.; Lagarce, F.; Garcion, E.; Benoit, J. P. Biopharmaceutical parameters to consider in order to alter the fate of nanocarriers after oral delivery. Nanomedicine 2010, 5, 287-306.

[14] Chao, P. Y.; Deshmukh, M.; Kutscher, H. L.; Gao, D. Y.; Rajan, S. S.; Hu, P. D.; Laskin, D. L.; Stein, S.; Sinko, P. J. Pulmonary targeting microparticulate camptothecin delivery system: Anticancer evaluation in a rat orthotopic lung cancer model. Anti-Cancer Drugs 2010, 21, 65-76.

[15] Patton, J. S.; Fishburn, C. S.; Weers, J. G. The lungs as a portal of entry for systemic drug delivery. Proc. Am. Thorac. Soc. 2004, 1, 338-344.

[16] Sung, J. C.; Pulliam, B. L.; Edwards, D. A. Nanoparticles for drug delivery to the lungs. Trends Biotechnol. 2007, 25, 563-570.

[17] Dailey, L. A.; Schmehl, T.; Gessler, T.; Wittmar, M.; Grimminger, F.; Seeger, W.; Kissel, T. Nebulization of biodegradable nanoparticles: Impact of nebulizer technology and nanoparticle characteristics on aerosol features. J. Controlled Release 2003, 86, 131-144.

[18] Geller, D. E.; Pitlick, W. H.; Nardella, P. A.; Tracewell, W. G.; Ramsey, B. W. Pharmacokinetics and bioavailability of aerosolized tobramycin in cystic fibrosis. Chest 2002, 122, 219-226.

[19] Flume, P. A.; VanDevanter, D. R. Clinical applications of pulmonary delivery of antibiotics. Adv. Drug Deliv. Rev. 2015, 85, 1-6.

[20] Wenzler, E.; Fraidenburg, D. R.; Scardina, T.; Danziger, L. H. Inhaled antibiotics for gram-negative respiratory infections. Clin. Microbiol. Rev. 2016, 29, 581-632. 
[21] Langton Hewer, S. C.; Smyth, A. R. Antibiotic strategies for eradicating Pseudomonas aeruginosa in people with cystic fibrosis. Cochrane Database. Syst. Rev. 2017, 4, CD004197.

[22] Cohen-Cymberknoh, M.; Shoseyov, D.; Kerem, E. Managing cystic fibrosis: Strategies that increase life expectancy and improve quality of life. Am. J. Respir. Crit. Care Med. 2011, 183, 1463-1471.

[23] Mei, L.; Zhu, G. Z.; Qiu, L. P.; Wu, C. C.; Chen, H. P.; Liang, H.; Cansiz, S.; Lv, Y. F.; Zhang, X. B.; Tan, W. H. Self-assembled multifunctional DNA nanoflowers for the circumvention of multidrug resistance in targeted anticancer drug delivery. Nano Res. 2015, 8 , 3447-3460.

[24] Zhou, Q.; Leung, S. S. Y.; Tang, P.; Parumasivam, T.; Loh, Z. H.; Chan, H. K. Inhaled formulations and pulmonary drug delivery systems for respiratory infections. Adv. Drug Deliv. Rev. 2015, 85, 83-99.

[25] Pérez, B. F.; Méndez, G. A.; Lagos, R. A.; Vargas, M. S. L. Mucociliary clearance system in lung defense. Rev. Med. Chil. 2014, 142, 606615.

[26] Hardy, C. L.; LeMasurier, J. S.; Mohamud, R.; Yao, J.; Xiang, S. D.; Rolland, J. M.; O'Hehir, R. E.; Plebanski, M. Differential uptake of nanoparticles and microparticles by pulmonary APC subsets induces discrete immunological imprints. J. Immunol. 2013, 191, 5278-5290.

[27] d'Angelo, I.; Conte, C.; La Rotonda, M. I.; Miro, A.; Quaglia, F.; Ungaro, F. Improving the efficacy of inhaled drugs in cystic fibrosis: Challenges and emerging drug delivery strategies. Adv. Drug Deliv. Rev. 2014, 75, 92-111.

[28] Hoffmann, I. M.; Rubin, B. K.; Iskandar, S. S.; Schechter, M. S.; Nagaraj, S. K.; Bitzan, M. M. Acute renal failure in cystic fibrosis: Association with inhaled tobramycin therapy. Pediatr. Pulm. 2002, 34, 375-377.

[29] Tolker-Nielsen, T. Pseudomonas aeruginosa biofilm infections: From molecular biofilm biology to new treatment possibilities. APMIS 2014, 122, 1-51.

[30] Allen, T. M.; Cullis, P. R. Drug delivery systems: Entering the mainstream. Science 2004, 303, 1818-1822.

[31] Koul, A.; Arnoult, E.; Lounis, N.; Guillemont, J.; Andries, K. The challenge of new drug discovery for tuberculosis. Nature 2011, 469, 483-490.

[32] Raeburn, D.; Underwood, S. L.; Villamil, M. E. Techniques for drug delivery to the airways, and the assessment of lung function in animal models. J. Pharmacol. Toxicol. Methods 1992, 27, 143-159.

[33] Thompson, D. C. J. D. Pharmacology of therapeutic aerosols. Science 1992, 54, 29-59.

[34] Courrier, H. M.; Butz, N.; Vandamme, T. F. Pulmonary drug delivery systems: Recent developments and prospects. Crit. Rev. Ther. Drug Carrier Syst. 2002, 19, 425-498.

[35] Clark, A. J. D. D. S. Sciences formulation of proteins and peptides for inhalation. Science 2002, 2, 73-77.

[36] Gill, S.; Löbenberg, R.; Ku, T.; Azarmi, S.; Roa, W.; Prenner, E. J. Nanoparticles: Characteristics, mechanisms of action, and toxicity in pulmonary drug delivery-a review. J. Biomed. Nanotechnol. 2007, 3, 107-119.

[37] Ranney, D. F. Drug targeting to the lungs. Biochem. Pharmacol. 1986, 35, 1063-1069.

[38] Barnes, P. J. Nocturnal asthma: Mechanisms and treatment. Br. Med. J. 1984, 288, 1397-1398.

[39] Surendrakumar, K.; Martyn, G. P.; Hodgers, E. C. M.; Jansen, M.; Blair, J. A. Sustained release of insulin from sodium hyaluronate based dry powder formulations after pulmonary delivery to beagle dogs. J. Control. Release 2003, 91, 385-394.

[40] Cook, R. O.; Pannu, R. K.; Kellaway, I. W. Novel sustained release microspheres for pulmonary drug delivery. J. Control. Release 2005, 104, 79-90.

[41] Hardy, J. G.; Chadwick, T. S. Sustained release drug delivery to the lungs: An option for the future. Clin. Pharmacokinet. 2000, 39, 1-4.

[42] Zeng, X. M.; Martin, G. P.; Marriott, C. The controlled delivery of drugs to the lung. Int. J. Pharm. 1995, 124, 149-164.

[43] Bourzac, K. Nanotechnology: Carrying drugs. Nature 2012, 491, S58-S60.

[44] Pelaz, B.; Alexiou, C.; Alvarez-Puebla, R. A.; Alves, F.; Andrews, A. M.; Ashraf, S.; Balogh, L. P.; Ballerini, L.; Bestetti, A.; Brendel, C. et al. Diverse applications of nanomedicine. ACS Nano 2017, 11, 2313-2381.
[45] Vij, N. Nano-based rescue of dysfunctional autophagy in chronic obstructive lung diseases. Expert Opin. Drug Deliv. 2017, 14, 483-489.

[46] Zhong, W.; Zhang, X.; Zhao, M.; Wu, J.; Lin, D. Advancements in nanotechnology for the diagnosis and treatment of multiple myeloma. Biomater. Sci. 2020, 8, 4692-4711.

[47] Chen, G. Y.; Roy, I.; Yang, C. H.; Prasad, P. N. Nanochemistry and nanomedicine for nanoparticle-based diagnostics and therapy. Chem. Rev. 2016, 116, 2826-2885.

[48] Singh, A. P.; Biswas, A.; Shukla, A.; Maiti, P. Targeted therapy in chronic diseases using nanomaterial-based drug delivery vehicles. Signal Transduct. Target. Ther. 2019, 4, 33.

[49] Xu, C. C.; Li, H.; Zhang, K. M.; Binzel, D. W.; Yin, H. R.; Chiu, W.; Guo, P. X. Photo-controlled release of paclitaxel and model drugs from RNA pyramids. Nano Res. 2019, 12, 41-48.

[50] Ma, Q. L.; Fan, Q.; Xu, J. L.; Bai, J. Y.; Han, X.; Dong, Z. L.; Zhou, X. Z.; Liu, Z.; Gu, Z.; Wang, C. Calming cytokine storm in pneumonia by targeted delivery of TPCA-1 using platelet-derived extracellular vesicles. Matter 2020, 3, 287-301.

[51] Zhang, X. Y.; Zhao, M. Y.; Cao, N.; Qin, W.; Zhao, M.; Wu, J.; Lin, D. J. Construction of a tumor microenvironment $\mathrm{pH}$-responsive cleavable PEGylated hyaluronic acid nano-drug delivery system for colorectal cancer treatment. Biomater. Sci. 2020, 8, 1885-1896.

[52] Wagner, V.; Dullaart, A.; Bock, A. K.; Zweck, A. The emerging nanomedicine landscape. Nat. Biotechnol. 2006, 24, 1211-1217.

[53] Stella, V. J.; Nti-Addae, K. W. Prodrug strategies to overcome poor water solubility. Adv. Drug Deliv. Rev. 2007, 59, 677-694.

[54] Savjani, K. T.; Gajjar, A. K.; Savjani, J. K. Drug solubility: Importance and enhancement techniques. Int. Scholar. Res. Not. 2012, 2012, 195727.

[55] De Jong, W. H.; Borm, P. J. A. Drug delivery and nanoparticles: Applications and hazards. Int. J. Nanomedicine 2008, 3, 133-149.

[56] Zhang, L.; Pornpattananangkul, D.; Hu, C. M. J.; Huang, C. M. Development of nanoparticles for antimicrobial drug delivery. Curr. Med. Chem. 2010, 17, 585-594.

[57] Murgia, X.; Loretz, B.; Hartwig, O.; Hittinger, M.; Lehr, C. M. The role of mucus on drug transport and its potential to affect therapeutic outcomes. Adv. Drug Deliv. Rev. 2018, 124, 82-97.

[58] Murgia, X.; Pawelzyk, P.; Schaefer, U. F.; Wagner, C.; Willenbacher, N.; Lehr, C. M. Size-limited penetration of nanoparticles into porcine respiratory mucus after aerosol deposition. Biomacromolecules 2016, 17, 1536-1542.

[59] Ho, D. K.; Costa, A.; De Rossi, C.; de Souza Carvalho-Wodarz, C.; Loretz, B.; Lehr, C. M. Polysaccharide submicrocarrier for improved pulmonary delivery of poorly soluble anti-infective ciprofloxacin: Preparation, characterization, and influence of size on cellular uptake. Mol. Pharmaceutics 2018, 15, 1081-1096.

[60] Markman, J. L.; Rekechenetskiy, A.; Holler, E.; Ljubimova, J. Y. Nanomedicine therapeutic approaches to overcome cancer drug resistance. Adv. Drug Deliv. Rev. 2013, 65, 1866-1879.

[61] Zhu, X.; Radovic-Moreno, A. F.; Wu, J.; Langer, R.; Shi, J. J. Nanomedicine in the management of microbial infection - overview and perspectives. Nano Today 2014, 9, 478-498.

[62] Abed, N.; Couvreur, P. Nanocarriers for antibiotics: A promising solution to treat intracellular bacterial infections. Int. J. Antimicrob. Agents 2014, 43, 485-496.

[63] Champion, J. A.; Katare, Y. K.; Mitragotri, S. Particle shape: A new design parameter for micro- and nanoscale drug delivery carriers. $J$. Controlled Release 2007, 121, 3-9.

[64] Gao, W. W.; Thamphiwatana, S.; Angsantikul, P.; Zhang, L. F. Nanoparticle approaches against bacterial infections. Wiley Interdiscip. Rev. Nanomed. Nanobiotechnol. 2014, 6, 532-547.

[65] Siegel, R. L.; Miller, K. D.; Jemal, A. Cancer statistics, 2019. CA Cancer J. Clin. 2019, 69, 7-34.

[66] Barenholz, Y. Doxil ${ }^{\circledR}$--the first FDA-approved nano-drug: Lessons learned. J. Control. Release 2012, 160, 117-134.

[67] Numico, G.; Castiglione, F.; Granetto, C.; Garrone, O.; Mariani, G.; Di Costanzo, G.; La Ciura, P.; Gasco, M.; Ostellino, O.; Porcile, G. et al. Single-agent pegylated liposomal doxorubicin $\left(\right.$ Caelix $\left.^{\circledR}\right)$ in chemotherapy pretreated non-small cell lung cancer patients: A pilot trial. Lung Cancer 2002, 35, 59-64.

[68] Patlakas, G.; Bouros, D.; Tsantekidou-Pozova, S.; Koukourakis, M. I. Triplet chemotherapy with docetaxel, gemcitabine and liposomal 
doxorubicin, supported with subcutaneous amifostine and hemopoietic growth factors, in advanced non-small cell lung cancer. Anticancer Res. 2005, 25, 1427-1431.

[69] Leighl, N. B.; Goss, G. D.; Lopez, P. G.; Burkes, R. L.; Dancey, J. E.; Rahim, Y. H.; Rudinskas, L. C.; Pouliot, J. F.; Rodgers, A.; Pond, G. R. et al. Phase II study of pegylated liposomal doxorubicin $\mathrm{HCl}$ (Caelyx) in combination with cyclophosphamide and vincristine as second-line treatment of patients with small cell lung cancer. Lung Cancer 2006, 52, 327-332.

[70] Xu, C. N.; Wang, Y. B.; Guo, Z. P.; Chen, J.; Lin, L.; Wu, J. Y.; Tian, H. Y.; Chen, X. S. Pulmonary delivery by exploiting doxorubicin and cisplatin co-loaded nanoparticles for metastatic lung cancer therapy. J. Control. Release 2019, 295, 153-163.

[71] Hoch, U.; Staschen, C. M.; Johnson, R. K.; Eldon, M. A. Nonclinical pharmacokinetics and activity of etirinotecan pegol (NKTR-102), a long-acting topoisomerase 1 inhibitor, in multiple cancer models. Cancer Chemother. Pharm. 2014, 74, 1125-1137.

[72] Jameson, G. S.; Hamm, J. T.; Weiss, G. J.; Alemany, C.; Anthony, S.; Basche, M.; Ramanathan, R. K.; Borad, M. J.; Tibes, R.; Cohn, A. et al. A multicenter, phase I, dose-escalation study to assess the safety, tolerability, and pharmacokinetics of etirinotecan pegol in patients with refractory solid tumors. Clin. Cancer Res. 2013, 19, 268-278.

[73] Aggarwal, C.; Cohen, R. B.; Yu, E.; Hwang, W. T.; Bauml, J. M.; Alley, E.; Evans, T. L.; Langer, C. J. Etirinotecan pegol (NKTR-102) in third-line treatment of patients with metastatic or recurrent non-small-cell lung cancer: Results of a phase II study. Clin. Lung Cancer 2018, 19, 157-162.

[74] Zhang, H. J. Onivyde for the therapy of multiple solid tumors. OncoTargets Ther. 2016, 9, 3001-3007.

[75] Leonard, S.; Lee, H.; Klinz, S.; Paz, N.; Fitzgerald, J.; Hendriks, B. P1.07-006 Preclinical support for evaluation of irinotecan liposome injection (nal-IRI, MM-398) in small cell lung cancer: Topic: Drug treatment alone and in combination with radiotherapy. J. Thorac. Oncol. 2017, 12, S699.

[76] Landesman-Milo, D.; Ramishetti, S.; Peer, D. Nanomedicine as an emerging platform for metastatic lung cancer therapy. Cancer Metastasis Rev. 2015, 34, 291-301.

[77] Zhang, Y. F.; Huang, Y. X.; Li, S. Polymeric micelles: Nanocarriers for cancer-targeted drug delivery. AAPS PharmSciTech. 2014, 15, 862-871.

[78] Gupta, N.; Hatoum, H.; Dy, G. K. First line treatment of advanced non-small-cell lung cancer-specific focus on albumin bound paclitaxel. Int. J. Nanomedicine 2014, 9, 209-221.

[79] Pazdur, R. J. N. C. I. a. t. N. I. o. H. R. A. FDA approval for paclitaxel albumin-stabilized nanoparticle formulation. National Cancer Institute at the National Institutes of Health 2012, 24, 2013.

[80] Jiménez-López, J.; El-Hammadi, M. M.; Ortiz, R.; Cayero-Otero, M. D.; Cabeza, L.; Perazzoli, G.; Martin-Banderas, L.; Baeyens, J. M.; Prados, J.; Melguizo, C. A novel nanoformulation of PLGA with high non-ionic surfactant content improves in vitro and in vivo PTX activity against lung cancer. Pharmacol. Res. 2019, 141, 451-465.

[81] Yang, Y.; Huang, Z. W.; Li, J. Y.; Mo, Z. R.; Huang, Y.; Ma, C.; Wang, W. H.; Pan, X.; Wu, C. B. PLGA porous microspheres dry powders for codelivery of afatinib-loaded solid lipid nanoparticles and paclitaxel: Novel therapy for EGFR tyrosine kinase inhibitors resistant nonsmall cell lung cancer. Adv. Healthc. Mater. 2019, 8 , 1900965.

[82] Ormerod, M. G.; Orr, R. M.; Peacock, J. H. The role of apoptosis in cell killing by cisplatin: A flow cytometric study. Br. J. Cancer 1994, $69,93-100$.

[83] Sears, C. R.; Cooney, S. A.; Chin-Sinex, H.; Mendonca, M. S.; Turchi, J. J. DNA damage response (DDR) pathway engagement in cisplatin radiosensitization of non-small cell lung cancer. DNA Rep. 2016, 40, 35-46.

[84] Sun, M.; He, L.; Fan, Z.; Tang, R. P.; Du, J. Z. Effective treatment of drug-resistant lung cancer via a nanogel capable of reactivating cisplatin and enhancing early apoptosis. Biomaterials 2020, 257, 120252.

[85] Iyer, R.; Nguyen, T.; Padanilam, D.; Xu, C. C.; Saha, D.; Nguyen, K. T.; Hong, Y. Glutathione-responsive biodegradable polyurethane nanoparticles for lung cancer treatment. J. Control. Release 2020, $321,363-371$.
[86] Stathopoulos, G. P.; Boulikas, T. Lipoplatin formulation review article. J. Drug Deliv. 2012, 2012, 581363.

[87] Kaltsas, K.; Anevlavis, S.; Pataka, A.; Kouliatsis, G.; Pozova, S.; Bouros, D.; Froudarakis, M. A phase II trial of lipoplatin and gemcitabine in patients with NSCLC previously treated with platinum-based chemotherapy. Eur. Respir. Soc. 2016, 48, OA3344.

[88] Chan, M. H.; Huang, W. T.; Wang, J.; Liu, R. S.; Hsiao, M. Nextgeneration cancer-specific hybrid theranostic nanomaterials: MAGE-A3 NIR persistent luminescence nanoparticles conjugated to afatinib for in situ suppression of lung adenocarcinoma growth and metastasis. Adv. Sci. 2020, 7, 1903741.

[89] Fehrenbacher, L.; Spira, A.; Ballinger, M.; Kowanetz, M.; Vansteenkiste, J.; Mazieres, J.; Park, K.; Smith, D.; Artal-Cortes, A.; Lewanski, C. et al. Atezolizumab versus docetaxel for patients with previously treated non-small-cell lung cancer (POPLAR): A multicentre, open-label, phase 2 randomised controlled trial. Lancet 2016, 387, 1837-1846.

[90] Garon, E. B.; Rizvi, N. A.; Hui, R. N.; Leighl, N.; Balmanoukian, A. S.; Eder, J. P.; Patnaik, A.; Aggarwal, C.; Gubens, M.; Horn, L. et al. Pembrolizumab for the treatment of non-small-cell lung cancer. $N$. Engl. J. Med. 2015, 372, 2018-2028.

[91] Liu, B.; Cao, W.; Qiao, G. L.; Yao, S. Y.; Pan, S. J.; Wang, L. R.; Yue, C. X.; Ma, L. J.; Liu, Y. L.; Cui, D. X. Effects of gold nanoprismassisted human PD-L1 siRNA on both gene down-regulation and photothermal therapy on lung cancer. Acta Biomater. 2019, 99, 307-319.

[92] Duman, F. D.; Akkoc, Y.; Demirci, G.; Bavili, N.; Kiraz, A.; Gozuacik, D.; Acar, H. Y. Bypassing pro-survival and resistance mechanisms of autophagy in EGFR-positive lung cancer cells by targeted delivery of 5FU using theranostic $\mathrm{Ag}_{2} \mathrm{~S}$ quantum dots. J. Mater. Chem. B. 2019, 7, 7363-7376.

[93] Reda, M.; Ngamcherdtrakul, W.; Gu, S. D.; Bejan, D. S.; Siriwon, N.; Gray, J. W.; Yantasee, W. PLK1 and EGFR targeted nanoparticle as a radiation sensitizer for non-small cell lung cancer. Cancer Lett. 2019, 467, 9-18.

[94] Bregoli, L.; Movia, D.; Gavigan-Imedio, J. D.; Lysaght, J.; Reynolds, J.; Prina-Mello, A. Nanomedicine applied to translational oncology: A future perspective on cancer treatment. Nanomedicine 2016, 12, 81-103.

[95] Seidi, K.; Neubauer, H. A.; Moriggl, R.; Jahanban-Esfahlan, R.; Javaheri, T. Tumor target amplification: Implications for nano drug delivery systems. J. Control. Release 2018, 275, 142-161.

[96] Chang, S. S.; O'Keefe, D. S.; Bacich, D. J.; Reuter, V. E.; Heston, W. D. W.; Gaudin, P. B. Prostate-specific membrane antigen is produced in tumor-associated neovasculature. Clin. Cancer Res. 1999, 5, 2674-2681.

[97] Hrkach, J.; Von Hoff, D.; Mukkaram Ali, M.; Andrianova, E.; Auer, J.; Campbell, T.; De Witt, D.; Figa, M.; Figueiredo, M.; Horhota, A. et al. Preclinical development and clinical translation of a PSMA-targeted docetaxel nanoparticle with a differentiated pharmacological profile. Sci. Transl. Med. 2012, 4, 128 ra39.

[98] Von Hoff, D. D.; Mita, M. M.; Ramanathan, R. K.; Weiss, G. J.; Mita, A. C.; LoRusso, P. M.; Burris III, H. A.; Hart, L. L.; Low, S. C.; Parsons, D. M. et al. Phase I study of PSMA-targeted docetaxelcontaining nanoparticle BIND-014 in patients with advanced solid tumors. Clin. Cancer Res. 2016, 22, 3157-3163.

[99] Viteri, S.; Rosell, R. An innovative mesothelioma treatment based on miR-16 mimic loaded EGFR targeted minicells (TargomiRs). Transl. Lung Cancer Res. 2018, 7, S1-S4.

[100] Reid, G.; Kao, S. C.; Pavlakis, N.; Brahmbhatt, H.; MacDiarmid, J.; Clarke, S.; Boyer, M.; van Zandwijk, N. Clinical development of TargomiRs, a miRNA mimic-based treatment for patients with recurrent thoracic cancer. Epigenomics 2016, 8, 1079-1085.

[101] Kao, S. C.; Fulham, M.; Wong, K.; Cooper, W.; Brahmbhatt, H.; MacDiarmid, J.; Pattison, S.; Sagong, J. O.; Huynh, Y.; Leslie, F. et al. A significant metabolic and radiological response after a novel targeted MicroRNA-based treatment approach in malignant pleural mesothelioma. Am. J. Respir. Crit. Care Med. 2015, 191, 1467-1469.

[102] Moro, M.; Di Paolo, D.; Milione, M.; Centonze, G.; Bornaghi, V.; Borzi, C.; Gandellini, P.; Perri, P.; Pastorino, U.; Ponzoni, M. et al. Coated cationic lipid-nanoparticles entrapping miR-660 inhibit 
tumor growth in patient-derived xenografts lung cancer models. J. Control. Release 2019, 308, 44-56.

[103] Lu, C.; Stewart, D. J.; Lee, J. J.; Ji, L.; Ramesh, R.; Jayachandran, G.; Nunez, M. I.; Wistuba, I. I.; Erasmus, J. J.; Hicks, M. E. et al. Phase I clinical trial of systemically administered TUSC2(FUS1)nanoparticles mediating functional gene transfer in humans. PLoS One 2012, 7, e34833.

[104] Sharma, S.; Srivastava, M. K.; Harris-White, M.; Lee, J. M.; Dubinett, S. MUC1 peptide vaccine mediated antitumor activity in non-small cell lung cancer. Expert Opin. Biol. Ther. 2011, 11, 987-990.

[105] Butts, C.; Murray, N.; Maksymiuk, A.; Goss, G.; Marshall, E.; Soulières, D.; Cormier, Y.; Ellis, P.; Price, A.; Sawhney, R. et al. Randomized phase IIB trial of BLP25 liposome vaccine in stage IIIB and IV non-small-cell lung cancer. J. Clin. Oncol. 2005, 23, 6674-6681.

[106] Wu, Y. L.; Park, K.; Soo, R. A.; Sun, Y.; Tyroller, K.; Wages, D.; Ely, G.; Yang, J. C. H.; Mok, T. INSPIRE: A phase III study of the BLP25 liposome vaccine (L-BLP25) in Asian patients with unresectable stage III non-small cell lung cancer. BMC Cancer 2011, 11, 430 .

[107] Griesenbach, U.; Pytel, K. M.; Alton, E. W. F. W. Cystic fibrosis gene therapy in the UK and elsewhere. Hum. Gene Ther. 2015, 26, 266-275.

[108] Hardee, C. L.; Arévalo-Soliz, L. M.; Hornstein, B. D.; Zechiedrich, L. Advances in non-viral DNA vectors for gene therapy. Genes 2017, 8, 65 .

[109] Konstan, M. W.; Davis, P. B.; Wagener, J. S.; Hilliard, K. A.; Stern, R. C.; Milgram, L. J. H.; Kowalczyk, T. H.; Hyatt, S. L.; Fink, T. L.; Gedeon, C. R. et al. Compacted DNA nanoparticles administered to the nasal mucosa of cystic fibrosis subjects are safe and demonstrate partial to complete cystic fibrosis transmembrane regulator reconstitution. Hum. Gene Ther. 2004, 15, 1255-1269.

[110] McLachlan, G.; Davidson, H.; Holder, E.; Davies, L. A.; Pringle, I. A.; Sumner-Jones, S. G.; Baker, A.; Tennant, P.; Gordon, C.; Vrettou, C. et al. Pre-clinical evaluation of three non-viral gene transfer agents for cystic fibrosis after aerosol delivery to the ovine lung. Gene Ther. 2011, 18, 996-1005.

[111] Alton, E. W. F. W.; Boyd, A. C.; Cheng, S. H.; Davies, J. C.; Davies, L. A.; Dayan, A.; Gill, D. R.; Griesenbach, U.; Higgins, T.; Hyde, S. C. et al. Toxicology study assessing efficacy and safety of repeated administration of lipid/DNA complexes to mouse lung. Gene Ther. 2014, 21, 89-95.

[112] Alton, E. W. F. W.; Baker, A.; Baker, E.; Boyd, A. C.; Cheng, S. H.; Coles, R. L.; Collie, D. D. S.; Davidson, H.; Davies, J. C.; Gill, D. R. et al. The safety profile of a cationic lipid-mediated cystic fibrosis gene transfer agent following repeated monthly aerosol administration to sheep. Biomaterials 2013, 34, 10267-10277.

[113] Alton, E. W. F. W.; Boyd, A. C.; Cheng, S. H.; Cunningham, S.; Davies, J. C.; Gill, D. R.; Griesenbach, U.; Higgins, T.; Hyde, S. C.; Innes, J. A. et al. A randomised, double-blind, placebo-controlled phase IIB clinical trial of repeated application of gene therapy in patients with cystic fibrosis. Thorax 2013, 68, 1075-1077.

[114] Alton, E. W. F. W.; Armstrong, D. K.; Ashby, D.; Bayfield, K. J.; Bilton, D.; Bloomfield, E. V.; Boyd, A. C.; Brand, J.; Buchan, R.; Calcedo, R. et al. Repeated nebulisation of non-viral CFTR gene therapy in patients with cystic fibrosis: A randomised, double-blind, placebo-controlled, phase $2 \mathrm{~b}$ trial. Lancet Res. Med. 2015, 3, 684-691.

[115] Robinson, E.; MacDonald, K. D.; Slaughter, K.; McKinney, M.; Patel, S.; Sun, C.; Sahay, G. Lipid nanoparticle-delivered chemically modified mRNA restores chloride secretion in cystic fibrosis. Mol. Ther. 2018, 26, 2034-2046.

[116] Haque, A. K. M. A.; Dewerth, A.; Antony, J. S.; Riethmüller, J.; Schweizer, G. R.; Weinmann, P.; Latifi, N.; Yasar, H.; Pedemonte, N.; Sondo, E. et al. Chemically modified hCFTR mRNAs recuperate lung function in a mouse model of cystic fibrosis. Sci. Rep. 2018, 8,16776 .

[117] Tagalakis, A. D.; Munye, M. M.; Ivanova, R.; Chen, H. P.; Smith, C. M.; Aldossary, A. M.; Rosa, L. Z.; Moulding, D.; Barnes, J. L.; Kafetzis, K. N. et al. Effective silencing of ENaC by siRNA delivered with epithelial-targeted nanocomplexes in human cystic fibrosis cells and in mouse lung. Thorax 2018, 73, 847-856.

[118] Osman, G.; Rodriguez, J.; Chan, S. Y.; Chisholm, J.; Duncan, G.; Kim, N.; Tatler, A. L.; Shakesheff, K. M.; Hanes, J.; Suk, J. S. et al. PEGylated enhanced cell penetrating peptide nanoparticles for lung gene therapy. J. Control. Release 2018, 285, 35-45.

[119] Leal, J.; Peng, X. J.; Liu, X. Q.; Arasappan, D.; Wylie, D. C.; Schwartz, S. H.; Fullmer, J. J.; McWilliams, B. C.; Smyth, H. D. C.; Ghosh, D. Peptides as surface coatings of nanoparticles that penetrate human cystic fibrosis sputum and uniformly distribute in vivo following pulmonary delivery. J. Control. Release 2020, 322, $457-469$

[120] Garbuzenko, O. B.; Kbah, N.; Kuzmov, A.; Pogrebnyak, N.; Pozharov, V.; Minko, T. Inhalation treatment of cystic fibrosis with lumacaftor and ivacaftor co-delivered by nanostructured lipid carriers. J. Control. Release 2019, 296, 225-231.

[121] Paranjpe, M.; Müller-Goymann, C. C. Nanoparticle-mediated pulmonary drug delivery: A review. Int. J. Mol. Sci. 2014, 15, $5852-5873$.

[122] Moreno-Sastre, M.; Pastor, M.; Salomon, C. J.; Esquisabel, A.; Pedraz, J. L. Pulmonary drug delivery: A review on nanocarriers for antibacterial chemotherapy. J. Antimicrob. Chemother. 2015, 70, 2945-2955.

[123] Günday Türeli, N.; Torge, A.; Juntke, J.; Schwarz, B. C.; SchneiderDaum, N.; Türeli, A. E.; Lehr, C. M.; Schneider, M. Ciprofloxacinloaded PLGA nanoparticles against cystic fibrosis $P$. aeruginosa lung infections. Eur. J. Pharm. Biopharm. 2017, 117, 363-371.

[124] Ernst, J.; Klinger-Strobel, M.; Arnold, K.; Thamm, J.; Hartung, A.; Pletz, M. W.; Makarewicz, O.; Fischer, D. Polyester-based particles to overcome the obstacles of mucus and biofilms in the lung for tobramycin application under static and dynamic fluidic conditions. Eur. J. Pharm. Biopharm. 2018, 131, 120-129.

[125] Mirza, S.; Clay, R. D.; Koslow, M. A.; Scanlon, P. D. COPD guidelines: A Review of the 2018 GOLD Report. Mayo Clin. Proc. 2018, 93, 1488-1502.

[126] da Silva, A. L.; Cruz, F. F.; Rocco, P. R. M.; Morales, M. M. New perspectives in nanotherapeutics for chronic respiratory diseases. Biophys. Rev. 2017, 9, 793-803.

[127] Wu, L.; Shan, W.; Zhang, Z. R.; Huang, Y. Engineering nanomaterials to overcome the mucosal barrier by modulating surface properties. Adv. Drug Deliv. Rev. 2018, 124, 150-163.

[128] Ramos, F. L.; Krahnke, J. S.; Kim, V. Clinical issues of mucus accumulation in COPD. Int. J. Chron. Obstruct. Pulmon. Dis. 2014, 9, 139-150.

[129] Zhang, X.; Zhang, W. T.; Liu, L. Z.; Yang, M.; Huang, L. J.; Chen, K.; Wang, R.; Yang, B. W.; Zhang, D. D.; Wang, J. L. Antibioticloaded $\mathrm{MoS}_{2}$ nanosheets to combat bacterial resistance via biofilm inhibition. Nanotechnology 2017, 28, 225101.

[130] Dua, K.; Shukla, S. D.; Tekade, R. K.; Hansbro, P. M. Whether a novel drug delivery system can overcome the problem of biofilms in respiratory diseases? Drug Deliv. Transl. Res. 2017, 7, 179-187.

[131] Dua, K.; de Jesus Andreoli Pinto, T.; Chellappan, D. K.; Gupta, G.; Bebawy, M.; Hansbro, P. M. Advancements in nano drug delivery systems: A challenge for biofilms in respiratory diseases. Panminerva Med. 2018, 60, 35-36.

[132] Mamary, A. J.; Criner, G. J. Tiotropium bromide for chronic obstructive pulmonary disease. Expert Rev. Respir. Med. 2009, 3, 211-220.

[133] Quon, B. S.; Goss, C. H.; Ramsey, B. W. Inhaled antibiotics for lower airway infections. Ann. Am. Thorac. Soc. 2014, 11, 425-434.

[134] Varshosaz, J.; Ghaffari, S.; Mirshojaei, S. F.; Jafarian, A.; Atyabi, F.; Kobarfard, F.; Azarmi, S. Biodistribution of amikacin solid lipid nanoparticles after pulmonary delivery. BioMed Res. Int. 2013, $2013,136859$.

[135] Kato, H.; Hagihara, M.; Hirai, J.; Sakanashi, D.; Suematsu, H.; Nishiyama, N.; Koizumi, Y.; Yamagishi, Y.; Matsuura, K.; Mikamo, H. Evaluation of amikacin pharmacokinetics and pharmacodynamics for optimal initial dosing regimen. Drugs R\&D 2017, 17, 177-187.

[136] Li, Z. B.; Luo, G. H.; Hu, W. P.; Hua, J. L.; Geng, S. Y.; Chu, P. K.; Zhang, J.; Wang, H. Y.; Yu, X. F. Mediated drug release from nanovehicles by black phosphorus quantum dots for efficient therapy of chronic obstructive pulmonary disease. Angew. Chem., Int. Ed. 2020, doi: 10.1002/anie. 202008379. 
[137] Chikuma, K.; Arima, K.; Asaba, Y.; Kubota, R.; Asayama, S.; Sato, K.; Kawakami, H. The potential of lipid-polymer nanoparticles as epigenetic and ROS control approaches for COPD. Free Radical Res. 2019, doi: 10.1080/10715762.2019.1696965.

[138] Mohamed, A.; Kunda, N. K.; Ross, K.; Hutcheon, G. A.; Saleem, I. Y. Polymeric nanoparticles for the delivery of miRNA to treat chronic obstructive pulmonary disease (COPD). Eur. J. Pharm. Biopharm. 2019, 136, 1-8.

[139] Vij, N.; Min, T.; Marasigan, R.; Belcher, C. N.; Mazur, S.; Ding, H.; Yong, K. T.; Roy, I. Development of PEGylated PLGA nanoparticle for controlled and sustained drug delivery in cystic fibrosis. $J$. Nanobiotechnol. 2010, 8, 22.

[140] Roy, I.; Vij, N. Nanodelivery in airway diseases: Challenges and therapeutic applications. Nanomedicine 2010, 6, 237-244.

[141] Vij, N. Nano-based theranostics for chronic obstructive lung diseases: Challenges and therapeutic potential. Expert. Opin. Drug Deliv. 2011, 8, 1105-1109.

[142] Vij, N.; Min, T.; Bodas, M.; Gorde, A.; Roy, I. Neutrophil targeted nano-drug delivery system for chronic obstructive lung diseases. Nanomedicine 2016, 12, 2415-2427.

[143] Barnes, P. J.; Burney, P. G. J.; Silverman, E. K.; Celli, B. R.; Vestbo, J.; Wedzicha, J. A.; Wouters, E. F. Chronic obstructive pulmonary disease. Nat. Rev. Dis. Primers 2015, 1, 15076.

[144] Andreeva, E.; Pokhaznikova, M.; Lebedev, A.; Moiseeva, I.; Kuznetsova, O.; Degryse, J. M. Spirometry is not enough to diagnose COPD in epidemiological studies: A follow-up study. Npj Prim. Care Respir. Med. 2017, 27, 62.

[145] Fens, N.; Zwinderman, A. H.; van der Schee, M. P.; de Nijs, S. B.; Dijkers, E.; Roldaan, A. C.; Cheung, D.; Bel, E. H.; Sterk, P. J. Exhaled breath profiling enables discrimination of chronic obstructive pulmonary disease and asthma. Am. J. Respir. Crit. Care Med. 2009, 180, 1076-1082.

[146] Dragonieri, S.; Annema, J. T.; Schot, R.; van der Schee, M. P. C.; Spanevello, A.; Carratú, P.; Resta, O.; Rabe, K. F.; Sterk, P. J. An electronic nose in the discrimination of patients with non-small cell lung cancer and COPD. Lung Cancer 2009, 64, 166-170.

[147] Sibila, O.; Garcia-Bellmunt, L.; Giner, J.; Merino, J. L.; SuarezCuartin, G.; Torrego, A.; Solanes, I.; Castillo, D.; Valera, J. L.; Cosio, B. G. et al. Identification of airway bacterial colonization by an electronic nose in chronic obstructive pulmonary disease. Respir. Med. 2014, 108, 1608-1614.

[148] Anusha, S.; CP, B.; Mohan, C. D.; Mathai, J.; Rangappa, S.; Mohan, S.; Chandra; Paricharak, S.; Mervin, L.; Fuchs, J. E. et al. A nano-MgO and ionic liquid-catalyzed 'green' synthesis protocol for the development of adamantyl-imidazolo-thiadiazoles as antituberculosis agents targeting sterol 14 $\alpha$-demethylase (CYP51). PLoS One 2015, 10, e0139798.

[149] Sharma, R.; Kaur, A.; Sharma, A. K.; Dilbaghi, N.; Sharma, A. K. Nano-based anti-tubercular drug delivery and therapeutic interventions in tuberculosis. Curr. Drug Targets 2017, 18, 72-86.

[150] Nasiruddin, M.; Neyaz, M. K.; Das, S. Nanotechnology-based approach in tuberculosis treatment. Tuberc. Res Treat. 2017, 2017, 4920209.

[151] Shojaei, T. R.; Mohd Salleh, M. A.; Tabatabaei, M.; Ekrami, A.; Motallebi, R.; Rahmani-Cherati, T.; Hajalilou, A.; Jorfi, R. Development of sandwich-form biosensor to detect Mycobacterium tuberculosis complex in clinical sputum specimens. Braz. J. Infect. Dis. 2014, $18,600-608$.

[152] Wang, S. Q.; Inci, F.; De Libero, G.; Singhal, A.; Demirci, U. Point-of-care assays for tuberculosis: Role of nanotechnology/ microfluidics. Biotechnol. Adv. 2013, 31, 438-449.

[153] Yang, H.; Qin, L. H.; Wang, Y. L.; Zhang, B. B.; Liu, Z. H.; Ma, H.; Lu, J. M.; Huang, X. C.; Shi, D. L.; Hu, Z. Y. Detection of Mycobacterium tuberculosis based on $\mathrm{H}_{37} \mathrm{R}_{\mathrm{v}}$ binding peptides using surface functionalized magnetic microspheres coupled with quantum dots-a nano detection method for Mycobacterium tuberculosis. Int. J. Nanomed. 2015, 10, 77-88.

[154] Cadena, A. M.; Fortune, S. M.; Flynn, J. A. L. Heterogeneity in tuberculosis. Nat. Rev. Immunol. 2017, 17, 691-702.

[155] Ellis, T.; Chiappi, M.; García-Trenco, A.; Al-Ejji, M.; Sarkar, S.; Georgiou, T. K.; Shaffer, M. S. P.; Tetley, T. D.; Schwander, S.; Ryan, M. P. et al. Multimetallic microparticles increase the potency of rifampicin against intracellular Mycobacterium tuberculosis. ACS Nano 2018, 12, 5228-5240.

[156] Hwang, A. A.; Lee, B. Y.; Clemens, D. L.; Dillon, B. J.; Zink, J. I.; Horwitz, M. A. pH-responsive isoniazid-loaded nanoparticles markedly improve tuberculosis treatment in Mice. Small 2015, 11, 5066-5078.

[157] Machelart, A.; Salzano, G.; Li, X.; Demars, A.; Debrie, A. S.; Menendez-Miranda, M.; Pancani, E.; Jouny, S.; Hoffmann, E.; Deboosere, N. et al. Intrinsic antibacterial activity of nanoparticles made of $\beta$-cyclodextrins potentiates their effect as drug nanocarriers against tuberculosis. ACS Nano 2019, 13, 3992-4007.

[158] Pi, J.; Shen, L.; Yang, E. Z.; Shen, H. B.; Huang, D.; Wang, R.; Hu, C. M.; Jin, H.; Cai, H. H.; Cai, J. Y. et al. Macrophage-targeted isoniazid-selenium nanoparticles promote antimicrobial immunity and synergize bactericidal destruction of tuberculosis bacilli. Angew. Chem., Int. Ed. 2020, 59, 3226-3234.

[159] Welin, A.; Björnsdottir, H.; Winther, M.; Christenson, K.; Oprea, T.; Karlsson, A.; Forsman, H.; Dahlgren, C.; Bylund, J. CFP-10 from Mycobacterium tuberculosis selectively activates human neutrophils through a pertussis toxin-sensitive chemotactic receptor. Infect. Immun. 2015, 83, 205-213.

[160] Feng, T. T.; Shou, C. M.; Shen, L.; Qian, Y.; Wu, Z. G.; Fan, J.; Zhang, Y. Z.; Tang, Y. W.; Wu, N. P.; Lu, H. Z. et al. Novel monoclonal antibodies to ESAT-6 and CFP-10 antigens for ELISA-based diagnosis of pleural tuberculosis. Int. J. Tuberc. Lung Dis. 2011, 15, 804-810.

[161] Liu, C.; Lyon, C. J.; Bu, Y.; Deng, Z. A.; Walters, E.; Li, Y.; Zhang, L. Q.; Hesseling, A. C.; Graviss, E. A.; Hu, Y. Clinical evaluation of a blood assay to diagnose paucibacillary tuberculosis via bacterial antigens. Clin. Chem. 2018, 64, 791-800.

[162] Liu, C.; Zhao, Z.; Fan, J.; Lyon, C. J.; Wu, H. J.; Nedelkov, D.; Zelazny, A. M.; Olivier, K. N.; Cazares, L. H.; Holland, S. M. et al. Quantification of circulating Mycobacterium tuberculosis antigen peptides allows rapid diagnosis of active disease and treatment monitoring. Prac. Natl. Acad. Sci. USA 2017, 114, 3969-3974.

[163] Phan, L. M. T.; Rafique, R.; Baek, S. H.; Nguyen, T. P.; Park, K. Y.; Kim, E. B.; Kim, J. G.; Park, J. P.; Kailasa, S. K.; Kim, H. J. et al. Gold-copper nanoshell dot-blot immunoassay for naked-eye sensitive detection of tuberculosis specific CFP-10 antigen. Biosens. Bioelectron. 2018, 121, 111-117.

[164] Meers, P.; Neville, M.; Malinin, V.; Scotto, A. W.; Sardaryan, G.; Kurumunda, R.; Mackinson, C.; James, G.; Fisher, S.; Perkins, W. $\mathrm{R}$. Biofilm penetration, triggered release and in vivo activity of inhaled liposomal amikacin in chronic Pseudomonas aeruginosa lung infections. J. Antimicrob. Chemother. 2008, 61, 859-868.

[165] Ehsan, Z.; Clancy, J. P. Management of Pseudomonas aeruginosa infection in cystic fibrosis patients using inhaled antibiotics with a focus on nebulized liposomal amikacin. Future Microbiol. 2015, 10, 1901-1912.

[166] Waters, V.; Ratjen, F. Inhaled liposomal amikacin. Expert Rev. Respir. Med. 2014, 8, 401-409.

[167] Costa-Gouveia, J.; Pancani, E.; Jouny, S.; Machelart, A.; Delorme, V.; Salzano, G.; Iantomasi, R.; Piveteau, C.; Queval, C. J.; Song, O. R. et al. Combination therapy for tuberculosis treatment: Pulmonary administration of ethionamide and booster co-loaded nanoparticles. Sci. Rep. 2017, 7, 5390.

[168] Serisier, D. J.; Bilton, D.; De Soyza, A.; Thompson, P. J.; Kolbe, J.; Greville, H. W.; Cipolla, D.; Bruinenberg, P.; Gonda, I. Inhaled, dual release liposomal ciprofloxacin in non-cystic fibrosis bronchiectasis (ORBIT-2): A randomised, double-blind, placebo-controlled trial. Thorax 2013, 68, 812-817.

[169] Doroudian, M.; MacLoughlin, R.; Poynton, F.; Prina-Mello, A.; Donnelly, S. C. Nanotechnology based therapeutics for lung disease. Thorax 2019, 74, 965-976.

[170] Hamblin, K. A.; Wong, J. P.; Blanchard, J. D.; Atkins, H. S. The potential of liposome-encapsulated ciprofloxacin as a tularemia therapy. Front. Cell. Infect. Microbiol. 2014, 4, 79.

[171] Wong, J. P.; Yang, H. M.; Blasetti, K. L.; Schnell, G.; Conley, J.; Schofield, L. N. Liposome delivery of ciprofloxacin against intracellular Francisella tularensis infection. J. Control. Release $\mathbf{2 0 0 3}, 92,265-273$. 
[172] Haworth, C.; Wanner, A.; Froehlich, J.; O'Neal, T.; Davis, A.; Gonda, I.; O'Donnell, A. Inhaled liposomal ciprofloxacin in patients with bronchiectasis and chronic Pseudomonas aeruginosa infection: Results from two parallel phase III trials (ORBIT-3 and-4). Am. J. Respir. Crit. Care Med. 2017, 195, A7604

[173] Ho, D. K.; Murgia, X.; De Rossi, C.; Christmann, R.; Hüfner de Mello Martins, A. G.; Koch, M.; Andreas, A.; Herrmann, J.; Müller, R.; Empting, M. et al. Squalenyl hydrogen sulfate nanoparticles for simultaneous delivery of tobramycin and an alkylquinolone quorum sensing inhibitor enable the eradication of $P$. aeruginosa biofilm infections. Angew. Chem., Int. Ed. 2020, 59, 10292-10296.

[174] Gao, Y. F.; Wang, J.; Chai, M. Y.; Li, X.; Deng, Y. Y.; Jin, Q.; Ji, J. Size and charge adaptive clustered nanoparticles targeting the biofilm microenvironment for chronic lung infection management. ACS Nano 2020, 14, 5686-5699.

[175] Wang, Y.; Yuan, Q.; Feng, W.; Pu, W. D.; Ding, J.; Zhang, H. J.; Li, X. Y.; Yang, B.; Dai, Q.; Cheng, L. et al. Targeted delivery of antibiotics to the infected pulmonary tissues using ROS-responsive nanoparticles. J. Nanobiotechnol. 2019, 17, 103.

[176] Zhang, C. Y.; Lin, W. J.; Gao, J.; Shi, X. T.; Davaritouchaee, M.; Nielsen, A. E.; Mancini, R. J.; Wang, Z. J. pH-responsive nanoparticles targeted to lungs for improved therapy of acute lung inflammation/injury. ACS Appl. Mater. Interfaces 2019, 11, 1638016390.

[177] Shirkhani, K.; Teo, I.; Armstrong-James, D.; Shaunak, S. Nebulised amphotericin B-polymethacrylic acid nanoparticle prophylaxis prevents invasive aspergillosis. Nanomedicine 2015, 11, 1217-1226.

[178] Tang, Y. X.; Wu, S.; Lin, J. Q.; Cheng, L. T.; Zhou, J.; Xie, J.; Huang, K. X.; Wang, X. Y.; Yu, Y.; Chen, Z. B. et al. Nanoparticles targeted against cryptococcal pneumonia by interactions between chitosan and its peptide ligand. Nano Lett. 2018, 18, 6207-6213.

[179] Qiu, L. X.; Hu, B. C.; Chen, H. B.; Li, S. S.; Hu, Y. Q.; Zheng, Y.; Wu, X. X. Antifungal efficacy of itraconazole-loaded TPGS-b-(PCLran-PGA) nanoparticles. Int. J. Nanomedicine 2015, 10, 1415-1423.

[180] Wang, J.; Li, P. Y.; Yu, Y.; Fu, Y. H.; Jiang, H. Y.; Lu, M.; Sun, Z. P.; Jiang, S. B.; Lu, L.; Wu, M. X. Pulmonary surfactant-biomimetic nanoparticles potentiate heterosubtypic influenza immunity. Science 2020, 367, eaau0810.

[181] Sanchez-Guzman, D.; Le Guen, P.; Villeret, B.; Sola, N.; Le Borgne, R.; Guyard, A.; Kemmel, A.; Crestani, B.; Sallenave, J. M.; Garcia-Verdugo, I. Silver nanoparticle-adjuvanted vaccine protects against lethal influenza infection through inducing BALT and IgAmediated mucosal immunity. Biomaterials 2019, 217, 119308.

[182] Kim, H.; Park, M.; Hwang, J.; Kim, J. H.; Chung, D. R.; Lee, K. S.; Kang, M. Development of label-free colorimetric assay for MERS-CoV using gold nanoparticles. ACS Sens. 2019, 4, 1306-1312.

[183] Layqah, L. A.; Eissa, S. An electrochemical immunosensor for the corona virus associated with the Middle East respiratory syndrome using an array of gold nanoparticle-modified carbon electrodes. Microchim. Acta 2019, 186, 224.

[184] Holgate, S. T.; Wenzel, S.; Postma, D. S.; Weiss, S. T.; Renz, H.; Sly, P. D. Asthma. Nat. Rev. Dis. Primers 2015, 1, 15025.

[185] Huang, C. L.; Wang, Y. M.; Li, X. W.; Ren, L. L.; Zhao, J. P.; Hu, Y.; Zhang, L.; Fan, G. H.; Xu, J. Y.; Gu, X. Y. et al. Clinical features of patients infected with 2019 novel coronavirus in Wuhan, China. Lancet 2020, 395, 497-506.

[186] Wang, D. W.; Hu, B.; Hu, C.; Zhu, F. F.; Liu, X.; Zhang, J.; Wang, B. B.; Xiang, H.; Cheng, Z. S.; Xiong, Y. et al. Clinical characteristics of 138 hospitalized patients with 2019 novel coronavirus-infected pneumonia in Wuhan, China. JAMA 2020, 323, 1061-1069.

[187] Zhou, P.; Yang, X. L.; Wang, X. G.; Hu, B.; Zhang, L.; Zhang, W.; Si, H. R.; Zhu, Y.; Li, B.; Huang, C. L. et al. A pneumonia outbreak associated with a new coronavirus of probable bat origin. Nature 2020, 579, 270-273.

[188] Zhu, N.; Zhang, D. Y.; Wang, W. L.; Li, X. W.; Yang, B.; Song, J. D.; Zhao, X.; Huang, B. Y.; Shi, W. F.; Lu, R. J. et al. A novel coronavirus from patients with pneumonia in China, 2019. N. Engl. J. Med. 2020, 382, 727-733.

[189] Wu, F.; Zhao, S.; Yu, B.; Chen, Y. M.; Wang, W.; Song, Z. G.; Hu, Y.; Tao, Z. W.; Tian, J. H.; Pei, Y. Y. et al. A new coronavirus associated with human respiratory disease in China. Nature 2020, 579, 265-269.
[190] Udugama, B.; Kadhiresan, P.; Kozlowski, H. N.; Malekjahani, A.; Osborne, M.; Li, V. Y. C.; Chen, H. M.; Mubareka, S.; Gubbay, J. B.; Chan, W. C. W. Diagnosing COVID-19: The disease and tools for detection. ACS nano. 2020, 14, 3822-3835.

[191] Moitra, P.; Alafeef, M.; Dighe, K.; Frieman, M. B.; Pan, D. Selective naked-eye detection of SARS-CoV-2 mediated by $\mathrm{n}$ gene targeted antisense oligonucleotide capped plasmonic nanoparticles. ACS Nano 2020, 14, 7617-7627.

[192] Chen, Z. H.; Zhang, Z. G.; Zhai, X. M.; Li, Y. Y.; Lin, L.; Zhao, H.; Bian, L.; Li, P.; Yu, L.; Wu, Y. S. et al. Rapid and sensitive detection of anti-SARS-CoV-2 IgG, using lanthanide-doped nanoparticles-based lateral flow immunoassay. Anal. Chem. 2020, 92, 7226-7231.

[193] Yadavalli, T.; Shukla, D. Role of metal and metal oxide nanoparticles as diagnostic and therapeutic tools for highly prevalent viral infections. Nanomedicine 2017, 13, 219-230.

[194] Quesada-González, D.; Merkoçi, A. Nanomaterial-based devices for point-of-care diagnostic applications. Chem. Soc. Rev. 2018, 47, 4697-4709.

[195] Maltez-da Costa, M.; de la Escosura-Muñiz, A.; Nogués, C.; Barrios, L.; Ibáñez, E.; Merkoçi, A. Simple monitoring of cancer cells using nanoparticles. Nano Lett. 2012, 12, 4164-4171.

[196] Seo, G.; Lee, G.; Kim, M. J.; Baek, S. H.; Choi, M.; Ku, K. B.; Lee, C. S.; Jun, S.; Park, D.; Kim, H. G. et al. Rapid detection of COVID-19 causative virus (SARS-CoV-2) in human nasopharyngeal swab specimens using field-effect transistor-based biosensor. $A C S$ Nano 2020, 14, 5135-5142.

[197] Szunerits, S.; Barras, A.; Khanal, M.; Pagneux, Q.; Boukherroub, R. Nanostructures for the inhibition of viral infections. Molecules 2015, 20, 14051-14081.

[198] Fahmi, M. Z.; Sukmayani, W.; Khairunisa, S. Q.; Witaningrum, A. M.; Indriati, D. W.; Matondang, M. Q. Y.; Chang, J. Y.; Kotaki, T.; Kameoka, M. Design of boronic acid-attributed carbon dots on inhibits HIV-1 entry. RSC Adv. 2016, 6, 92996-93002.

[199] Łoczechin, A.; Séron, K.; Barras, A.; Giovanelli, E.; Belouzard, S.; Chen, Y. T.; Metzler-Nolte, N.; Boukherroub, R.; Dubuisson, J.; Szunerits, S. Functional carbon quantum dots as medical countermeasures to human coronavirus. ACS Appl. Mater. Interfaces 2019, 11, 42964-42974.

[200] Yuan, Y.; Cao, D. F.; Zhang, Y. F.; Ma, J.; Qi, J. X.; Wang, Q. H.; Lu, G. W.; Wu, Y.; Yan, J. H.; Shi, Y. et al. Cryo-EM structures of MERS-CoV and SARS-CoV spike glycoproteins reveal the dynamic receptor binding domains. Nat. Commun. 2017, 8, 15092.

[201] Raj, V. S.; Mou, H.; Smits, S. L.; Dekkers, D. H. W.; Müller, M. A.; Dijkman, R.; Muth, D.; Demmers, J. A. A.; Zaki, A.; Fouchier, R. A. M. et al. Dipeptidyl peptidase 4 is a functional receptor for the emerging human coronavirus-EMC. Nature 2013, 495, 251-254.

[202] Coleman, C. M.; Venkataraman, T.; Liu, Y. V.; Glenn, G. M.; Smith, G. E.; Flyer, D. C.; Frieman, M. B. MERS-CoV spike nanoparticles protect mice from MERS-CoV infection. Vaccine 2017, 35, 15861589.

[203] Shereen, M. A.; Khan, S.; Kazmi, A.; Bashir, N.; Siddique, R. COVID-19 infection: Origin, transmission, and characteristics of human coronaviruses. J. Adv. Res. 2020, 24, 91-98.

[204] Monteil, V.; Kwon, H.; Prado, P.; Hagelkrüys, A.; Wimmer, R. A.; Stahl, M.; Leopoldi, A.; Garreta, E.; Hurtado Del Pozo, C.; Prosper, F. et al. Inhibition of SARS-CoV-2 infections in engineered human tissues using clinical-grade soluble human ACE2. Cell 2020, 181, 905-913.e7.

[205] Zhang, H. B.; Penninger, J. M.; Li, Y. M.; Zhong, N. S.; Slutsky, A. S. Angiotensin-converting enzyme 2 (ACE2) as a SARS-CoV-2 receptor: Molecular mechanisms and potential therapeutic target. Intens. Care Med. 2020, 46, 586-590.

[206] Hoffmann, M.; Kleine-Weber, H.; Schroeder, S.; Krüger, N.; Herrler, T.; Erichsen, S.; Schiergens, T. S.; Herrler, G.; Wu, N. H.; Nitsche, A. et al. SARS-CoV-2 cell entry depends on ACE2 and TMPRSS2 and is blocked by a clinically proven protease inhibitor. Cell 2020, 181, 271-280.e8.

[207] Singh, L.; Kruger, H. G.; Maguire, G. E. M.; Govender, T.; Parboosing, R. The role of nanotechnology in the treatment of viral infections. Ther. Adv. Infect. Dis. 2017, 4, 105-131.

[208] Jackman, J. A.; Lee, J.; Cho, N. J. Nanomedicine for infectious disease applications: Innovation towards broad-spectrum treatment 
of viral infections. Small 2016, 12, 1133-1139.

[209] Adesina, S. K.; Akala, E. O. Nanotechnology approaches for the delivery of exogenous siRNA for HIV therapy. Mol. Pharmaceutics 2015, 12, 4175-4187.

[210] Richardson, P.; Griffin, I.; Tucker, C.; Smith, D.; Oechsle, O.; Phelan, A.; Rawling, M.; Savory, E.; Stebbing, J. Baricitinib as potential treatment for $2019-\mathrm{nCoV}$ acute respiratory disease. Lancet $\mathbf{2 0 2 0}$, 395, e30-e31.

[211] Pu, S. Y.; Xiao, F.; Schor, S.; Bekerman, E.; Zanini, F.; BarouchBentov, R.; Nagamine, C. M.; Einav, S. Feasibility and biological rationale of repurposing sunitinib and erlotinib for dengue treatment. Antiviral. Res. 2018, 155, 67-75.

[212] Chen, N. S.; Zhou, M.; Dong, X.; Qu, J. M.; Gong, F. Y.; Han, Y.; Qiu, Y.; Wang, J. L.; Liu, Y.; Wei, Y. et al. Epidemiological and clinical characteristics of 99 cases of 2019 novel coronavirus pneumonia in Wuhan, China: A descriptive study. Lancet 2020, 395, 507-513.

[213] Xu, H. P.; He, C. Y.; Liu, Y.; Jiang, J. L.; Ma, T. Novel therapeutic modalities and drug delivery-erlotinib liposomes modified with galactosylated lipid: In vitro and in vivo investigations. Artif. Cells Nanomed. Biotechnol. 2018, 46, 1902-1907.

[214] Zeng, C. X.; Hou, X. C.; Yan, J. Y.; Zhang, C. X.; Li, W. Q.; Zhao, W. Y.; Du, S.; Dong, Y. Z. Leveraging mRNAs sequences to express SARS-CoV-2 antigens in vivo. bioRxiv 2020, doi: 10.1101/2020.04.01.019877.

[215] Broza, Y. Y.; Haick, H. Nanomaterial-based sensors for detection of disease by volatile organic compounds. Nanomedicine 2013, 8 , 785-806.

[216] Dragonieri, S.; Schot, R.; Mertens, B. J. A.; Le Cessie, S.; Gauw, S. A.; Spanevello, A.; Resta, O.; Willard, N. P.; Vink, T. J.; Rabe, K. F. et al. An electronic nose in the discrimination of patients with asthma and controls. J. Allergy Clin. Immunol. 2007, 120, 856-862.

[217] Taylor, S. L.; Leong, L. E. X.; Choo, J. M.; Wesselingh, S.; Yang, I. A.; Upham, J. W.; Reynolds, P. N.; Hodge, S.; James, A. L.; Jenkins, C. et al. Inflammatory phenotypes in patients with severe asthma are associated with distinct airway microbiology. J. Allergy Clin. Immunol. 2018, 141, 94-103.e15.

[218] Plaza, V.; Crespo, A.; Giner, J.; Merino, J. L.; Ramos-Barbón, D.; Mateus, E. F.; Torrego, A.; Cosio, B. G.; Agustí, A.; Sibila, O. Inflammatory asthma phenotype discrimination using an electronic nose breath analyzer. J. Investig. Allergol. Clin. Immunol. 2015, $25,431-437$.

[219] Keil, T. W. M.; Feldmann, D. P.; Costabile, G.; Zhong, Q.; da Rocha, S.; Merkel, O. M. Characterization of spray dried powders with nucleic acid-containing PEI nanoparticles. Eur. J. Pharm. Biopharm. 2019, 143, 61-69.

[220] Bhavna; Ahmad, F. J.; Mittal, G.; Jain, G. K.; Malhotra, G.; Khar, R. K.; Bhatnagar, A. Nano-salbutamol dry powder inhalation: A new approach for treating broncho-constrictive conditions. Eur. J. Pharm. Biopharm. 2009, 71, 282-291.

[221] Matsuo, Y.; Ishihara, T.; Ishizaki, J.; Miyamoto, K. I.; Higaki, M.; Yamashita, N. Effect of betamethasone phosphate loaded polymeric nanoparticles on a murine asthma model. Cell. Immunol. 2009, 260,33-38.

[222] Nasr, M.; Najlah, M.; D'Emanuele, A.; Elhissi, A. PAMAM dendrimers as aerosol drug nanocarriers for pulmonary delivery via nebulization. Int. J. Pharmaceut. 2014, 461, 242-250.

[223] Kenyon, N. J.; Bratt, J. M.; Lee, J.; Luo, J. T.; Franzi, L. M.; Zeki, A. A.; Lam, K. S. Self-assembling nanoparticles containing dexamethasone as a novel therapy in allergic airways inflammation. PLoS One 2013, 8, e77730.

[224] Chen, X. Y.; Huang, W. H.; Wong, B. C.; Yin, L. L.; Wong, Y. F.; $\mathrm{Xu}, \mathrm{M}$.; Yang, Z. J. Liposomes prolong the therapeutic effect of anti-asthmatic medication via pulmonary delivery. Int. J. Nanomedicine 2012, 7, 1139-1148.

[225] Arafa, M. G.; Ayoub, B. M. Nano-vesicles of salbutamol sulphate in metered dose inhalers: Formulation, characterization and in vitro evaluation. Int. J. Appl. Pharmaceut. 2017, 9, 100-105.

[226] Wang, K.; Feng, Y. P.; Li, S.; Li, W. J.; Chen, X.; Yi, R.; Zhang, H. R.; Hong, Z. Y. Oral delivery of bavachinin-loaded PEG-PLGA nanoparticles for asthma treatment in a murine model. J. Biomed. Nanotechnol. 2018, 14, 1806-1815.

[227] Chakraborty, S.; Ehsan, I.; Mukherjee, B.; Mondal, L.; Roy, S.; Saha, K. D.; Paul, B.; Debnath, M. C.; Bera, T. Therapeutic potential of andrographolide-loaded nanoparticles on a murine asthma model. Nanomedicine 2019, 20, 102006.

[228] Joshi, V. B.; Adamcakova-Dodd, A.; Jing, X. F.; Wongrakpanich, A.; Gibson-Corley, K. N.; Thorne, P. S.; Salem, A. K. Development of a poly (lactic-co-glycolic acid) particle vaccine to protect against house dust mite induced allergy. AAPS J. 2014, 16, 975-985.

[229] Salem, A. K. A promising CpG adjuvant-loaded nanoparticle-based vaccine for treatment of dust mite allergies. Immunotherapy 2014, 6, 1161-1163.

[230] Grozdanovic, M.; Laffey, K. G.; Abdelkarim, H.; Hitchinson, B.; Harijith, A.; Moon, H. G.; Park, G. Y.; Rousslang, L. K.; Masterson, J. C.; Furuta, G. T. et al. Novel peptide nanoparticle-biased antagonist of CCR3 blocks eosinophil recruitment and airway hyperresponsiveness. J. Allergy Clin. Immunol. 2019, 143, 669680. e612.

[231] Kumar, M.; Kong, X. Y.; Behera, A. K.; Hellermann, G. R.; Lockey, R. F.; Mohapatra, S. S. Chitosan IFN- $\gamma-$ pDNA nanoparticle (CIN) therapy for allergic asthma. Genet. Vaccines Ther. 2003, $1,3$.

[232] Kong, X. Y.; Hellermann, G. R.; Zhang, W. D.; Jena, P.; Kumar, M.; Behera, A.; Behera, S.; Lockey, R.; Mohapatra, S. S. Chitosan interferon- $\gamma$ nanogene therapy for lung disease: Modulation of T-cell and dendritic cell immune responses. Allergy Asthma Clin. Immnuol. 2008, 4, 95-105.

[233] Farokhzad, O. C.; Langer, R. Impact of nanotechnology on drug delivery. ACS Nano 2009, 3, 16-20.

[234] Shi, J. J.; Votruba, A. R.; Farokhzad, O. C.; Langer, R. Nanotechnology in drug delivery and tissue engineering: From discovery to applications. Nano Lett. 2010, 10, 3223-3230.

[235] Sanhai, W. R.; Sakamoto, J. H.; Canady, R.; Ferrari, M. Seven challenges for nanomedicine. Nat. Nanotechnol. 2008, 3, 242-244.

[236] Sanders, N.; Rudolph, C.; Braeckmans, K.; De Smedt, S. C.; Demeester, J. Extracellular barriers in respiratory gene therapy. Adv. Drug Deliv. Rev. 2009, 61, 115-127.

[237] Lai, S. K.; Wang, Y. Y.; Hanes, J. Mucus-penetrating nanoparticles for drug and gene delivery to mucosal tissues. Adv. Drug Deliv. Rev. 2009, 61, 158-171.

[238] Lai, S. K.; Wang, Y. Y.; Wirtz, D.; Hanes, J. Micro- and macrorheology of mucus. Adv. Drug Deliv. Rev. 2009, 61, 86-100.

[239] Petros, R. A.; DeSimone, J. M. Strategies in the design of nanoparticles for therapeutic applications. Nat. Rev. Drug Discov. 2010, 9, 615-627.

[240] Dobrovolskaia, M. A.; McNeil, S. E. Immunological properties of engineered nanomaterials. Nat Nanotechnol. 2007, 2, 469-478.

[241] Schuster, B. S.; Suk, J. S.; Woodworth, G. F.; Hanes, J. Nanoparticle diffusion in respiratory mucus from humans without lung disease. Biomaterials 2013, 34, 3439-3446.

[242] Huckaby, J. T.; Lai, S. K. PEGylation for enhancing nanoparticle diffusion in mucus. Adv. Drug Deliv. Rev. 2018, 124, 125-139.

[243] Shi, J. J.; Kantoff, P. W.; Wooster, R.; Farokhzad, O. C. Cancer nanomedicine: Progress, challenges and opportunities. Nat. Rev. Cancer 2017, 17, 20-37.

[244] Hare, J. I.; Lammers, T.; Ashford, M. B.; Puri, S.; Storm, G.; Barry, S. T. Challenges and strategies in anti-cancer nanomedicine development: An industry perspective. Adv. Drug Deliv. Rev. 2017, $108,25-38$.

[245] Kuzmov, A.; Minko, T. Nanotechnology approaches for inhalation treatment of lung diseases. J. Control. Release 2015, 219, 500-518.

[246] Hossen, S.; Hossain, M. K.; Basher, M. K.; Mia, M. N. H.; Rahman, M. T.; Uddin, M. J. Smart nanocarrier-based drug delivery systems for cancer therapy and toxicity studies: A review. J. Adv. Res. 2019, $15,1-18$.

[247] Wilhelm, S.; Tavares, A. J.; Dai, Q.; Ohta, S.; Audet, J.; Dvorak, H. F.; Chan, W. C. W. Analysis of nanoparticle delivery to tumours. Nat. Rev. Mater. 2016, $1,16014$.

[248] Genchi, G. G.; Marino, A.; Tapeinos, C.; Ciofani, G. Smart materials meet multifunctional biomedical devices: Current and prospective implications for nanomedicine. Front. Bioeng. Biotechnol. 2017, 5, 80. 